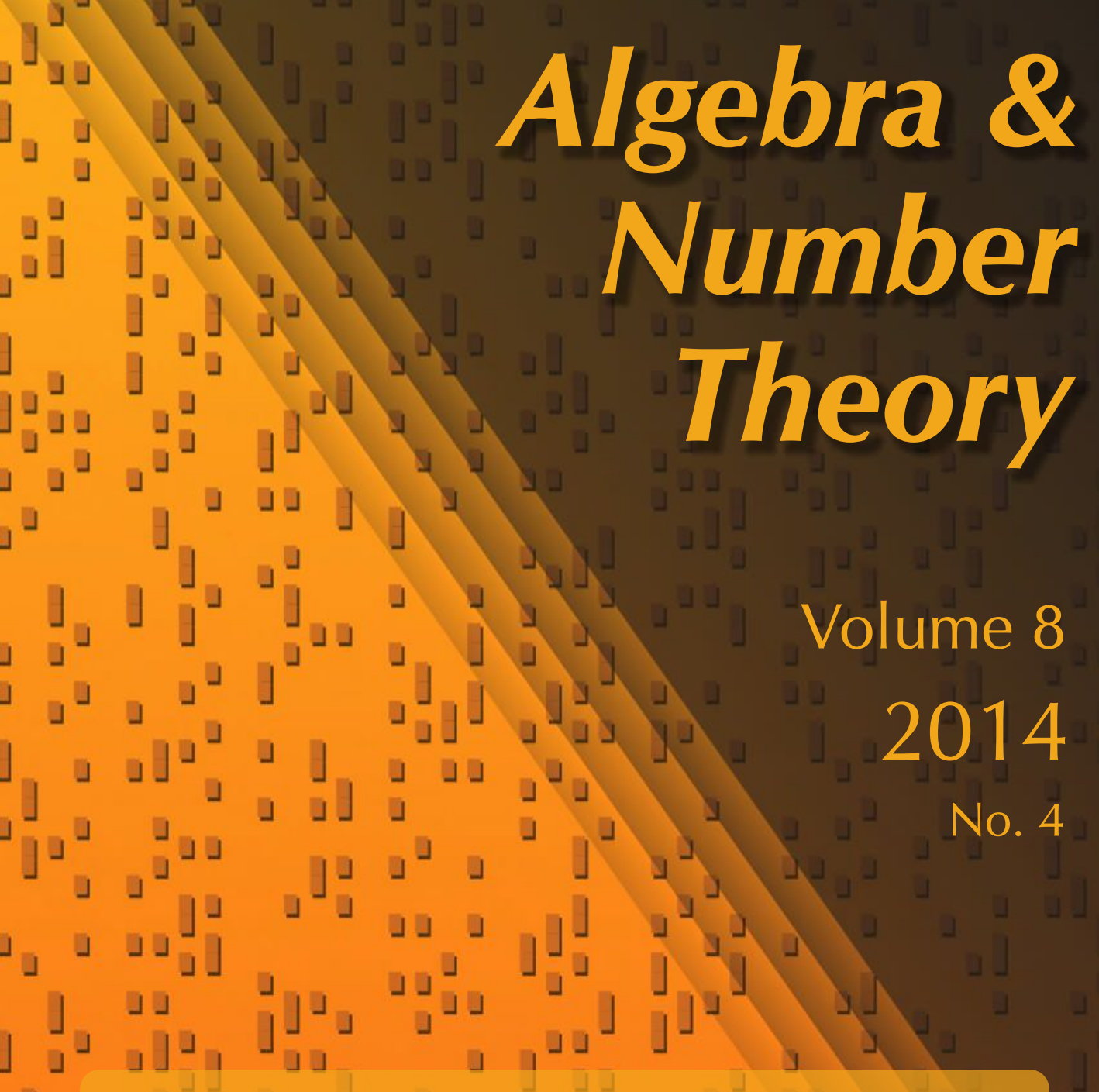

Posets, tensor products and Schur positivity

Vyjayanthi Chari, Ghislain Fourier and Daisuke Sagaki

\lrcorner

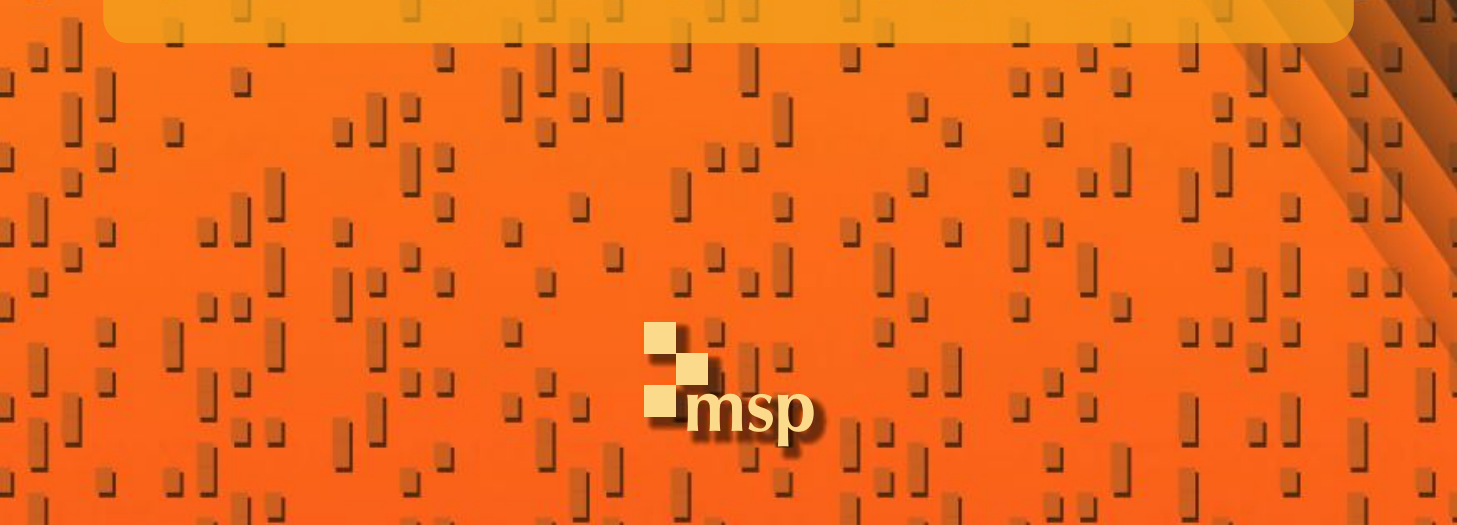




\title{
Posets, tensor products and Schur positivity
}

\author{
Vyjayanthi Chari, Ghislain Fourier and Daisuke Sagaki
}

Let $\mathfrak{g}$ be a complex finite-dimensional simple Lie algebra. Given a positive integer $k$ and a dominant weight $\lambda$, we define a preorder $\preceq$ on the set $P^{+}(\lambda, k)$ of $k$-tuples of dominant weights which add up to $\lambda$. Let $\sim$ be the equivalence relation defined by the preorder and $P^{+}(\lambda, k) / \sim$ be the corresponding poset of equivalence classes. We show that if $\lambda$ is a multiple of a fundamental weight (and $k$ is general) or if $k=2$ (and $\lambda$ is general), then $P^{+}(\lambda, k) / \sim$ coincides with the set of $S_{k}$-orbits in $P^{+}(\lambda, k)$, where $S_{k}$ acts on $P^{+}(\lambda, k)$ as the permutations of components. If $\mathfrak{g}$ is of type $A_{n}$ and $k=2$, we show that the $S_{2}$-orbit of the row shuffle defined by Fomin et al. (2005) is the unique maximal element in the poset.

Given an element of $P^{+}(\lambda, k)$, consider the tensor product of the corresponding simple finite-dimensional $\mathfrak{g}$-modules. We show that (for general $\mathfrak{g}, \lambda$, and $k$ ) the dimension of this tensor product increases along $\preceq$. We also show that in the case when $\lambda$ is a multiple of a fundamental minuscule weight ( $\mathfrak{g}$ and $k$ are general) or if $\mathfrak{g}$ is of type $A_{2}$ and $k=2$ ( $\lambda$ is general), there exists an inclusion of tensor products along with the partial order $\preceq$ on $P^{+}(\lambda, k) / \sim$. In particular, if $\mathfrak{g}$ is of type $A_{n}$, this means that the difference of the characters is Schur positive.

\section{Introduction}

This paper is partially motivated by the following simple observation. The isomorphism classes of simple finite-dimensional representations of the complex simple Lie algebra $\mathfrak{s l}_{2}$ are indexed by $\mathbb{Z}_{+}$, the set of nonnegative integers. Given $r \in \mathbb{Z}_{+}$, let $V(r)$ be a representative of the corresponding isomorphism class. The well-known Clebsch-Gordan formula gives us the following decomposition: for $r, s \in \mathbb{Z}_{+}$,

$$
V(r) \otimes V(s) \cong V(r+s) \oplus V(r+s-2) \oplus \cdots \oplus V(|r-s|) .
$$

Chari was partially supported by DMS-0901253. Fourier was partially supported by the DFG priority program 1388 "Representation Theory". Sagaki was partially supported by the Grant-in-Aid for Young Scientists (B) No. 23740003.

MSC2010: 17B67.

Keywords: Schur positivity, tensor products, posets, Lie algebras. 
If $r_{1}, s_{1} \in \mathbb{Z}_{+}$are such that $r_{1}+s_{1}=r+s$, it is then immediate that one has an injection of $\mathfrak{s l}_{2}$-modules

$$
V(r) \otimes V(s) \hookrightarrow V\left(r_{1}\right) \otimes V\left(s_{1}\right) \Longleftrightarrow|r-s| \geq\left|r_{1}-s_{1}\right| .
$$

In particular, the dimension increases if $\min \{r, s\} \leq \min \left\{r_{1}, s_{1}\right\}$. Moreover, the pairs $(r, r)$ and $(r, r+1)$ are maximal in the sense that: the corresponding tensor product maps onto to any tensor product corresponding to $\left(r_{1}, s_{1}\right)$ if $r_{1}+s_{1}=2 r$ (resp. $r_{1}+s_{1}=2 r+1$ ). Notice that these maximal pairs are actually the simplest examples of the row shuffle of partitions given in [Fomin et al. 2005]. Part of our interest in this problem comes from the fact that the tensor product $V(r) \otimes V(s)$ admits the structure of an indecomposable module for the infinite-dimensional Lie algebra $\mathfrak{s l}_{2} \otimes \mathbb{C}[t]$, where $\mathbb{C}[t]$ is the polynomial ring in the indeterminate $t$. In this case, the map in (0-1) is actually a map of $\mathfrak{s l}_{2} \otimes \mathbb{C}[t]$-modules and the module corresponding to the maximal pair is a truncation of a local Weyl module. We shall return to these ideas elsewhere. We mention this only to indicate our original motivation; the results of the current paper are entirely about simple Lie algebras.

Suppose now that $\mathfrak{g}$ is a finite-dimensional simple Lie algebra and $P^{+}$the set of dominant integral weights. We let $P^{+}(\lambda, 2)$ be the set of "compositions" of $\lambda$ with at most two parts, that is, pairs of dominant integral weights which add up to $\lambda$. If $\left(\lambda_{1}, \lambda_{2}\right)$ and $\left(\mu_{1}, \mu_{2}\right)$ are two such compositions, we define a partial order $\preceq$ by requiring that $\min \left\{\lambda_{1}\left(h_{\alpha}\right), \lambda_{2}\left(h_{\alpha}\right)\right\} \leq \min \left\{\mu_{1}\left(h_{\alpha}\right), \mu_{2}\left(h_{\alpha}\right)\right\}$ hold for all positive roots $\alpha$. This order extends in a natural way to $P^{+}(\lambda, k)$, the set of compositions of $\lambda$ with $k$ parts for all $k \geq 1$; one just requires the inequality to hold for all partial sums.

For $\mu \in P^{+}$, let $V(\mu)$ be the corresponding finite-dimensional $\mathfrak{g}$-module. If $\lambda$ and $\boldsymbol{\mu}$ are two compositions of $\lambda$ with $k$ parts and $V(\lambda), V(\boldsymbol{\mu})$ are the corresponding tensor products of $\mathfrak{g}$-modules, we prove that

$$
\boldsymbol{\lambda} \preceq \boldsymbol{\mu} \Longrightarrow \operatorname{dim} V(\boldsymbol{\lambda}) \leq \operatorname{dim} V(\boldsymbol{\mu}) .
$$

If $\lambda$ is a multiple of a minuscule weight, then we prove that there exists a (noncanonical) inclusion $V(\lambda) \hookrightarrow V(\boldsymbol{\mu})$. In the case when $\mathfrak{g}$ is of type $\mathfrak{s l}_{3}$ and $k=2$, we prove that the inclusion holds for all $\lambda \in P^{+}$. We conjecture that this latter result holds for all simple Lie algebras. Our conjecture may be viewed as a generalization of the row-shuffle conjecture which was made in [Fomin et al. 2005] for representations of $\mathfrak{s l}_{n+1}$. The row-shuffle conjecture was proved in [Lam et al. 2007], showing that our conjecture is true in the case of $\mathfrak{s l}_{n+1}$ for the pair $\boldsymbol{\lambda}, \boldsymbol{\mu}$ where $\boldsymbol{\mu}$ is the maximal element in the poset. A completely different approach taken in [Dobrovolska and Pylyavskyy 2007] also gives some evidence for our conjecture in the case of $\mathfrak{s l}_{n+1}$.

The following is an immediate combinatorial consequence of Theorem 1(i) and is perhaps of independent interest. Given two partitions $\left(0 \leq a_{1} \leq a_{2} \leq \cdots \leq a_{k}\right)$ 
and $\left(0 \leq b_{1} \leq b_{2} \leq \cdots \leq b_{k}\right)$ of an integer $n$ satisfying

$$
\sum_{j=1}^{i} a_{j} \leq \sum_{j=1}^{i} b_{j} \text { for all } 1 \leq i \leq k,
$$

we have

$$
a_{1} \ldots a_{k} \leq b_{1} \ldots b_{k}
$$

with equality if and only if $a_{j}=b_{j}$ for all $1 \leq j \leq k$.

The article is organized as follows. Section 1 has the basic definitions and notation. In Section 2 we introduce the partial order and state the main theorem. In Section 3 we prove that the dimension of the tensor product increases along the partial order. The critical idea in this proof is to show that in the case of $\mathfrak{s l}_{2}$, the partial order for $k>2$ is determined by the order at $k=2$. Once this is done, the proof is a simple application of the Weyl dimension formula. In Section 4 we use the results of Section 2 and the Littelmann path model to prove that for general $k$ and $\lambda$ a multiple of a minuscule weight, we have an inclusion of tensor products along the partial order. In Section 5 we study the partial order $P^{+}(\lambda, 2)$ in detail. We identify maximal elements of the poset and prove that the row shuffle is the unique maximal element when $\mathfrak{g}$ is of type $\mathfrak{s l}_{n+1}$. Finally, in Section 6 , we use results of Kashiwara and Nakashima on semistandard Young tableaux and crystal bases to prove that in the case that $\mathfrak{g}=\mathfrak{s l}_{3}$ and $k=2$, the Schur positivity holds along our order for all $\lambda \in P^{+}$.

\section{Preliminaries}

Throughout this paper we denote by $\mathbb{C}$ the field of complex numbers and by $\mathbb{Z}$ (resp. $\mathbb{Z}_{+}$) the set of integers (resp. nonnegative integers).

1.1. Let $\mathfrak{g}$ denote a finite-dimensional complex simple Lie algebra of rank $n$ and $\mathfrak{h}$ a fixed Cartan subalgebra of $\mathfrak{g}$. Let $I=\{1, \ldots, n\}$ and fix a set $\left\{\alpha_{i}: i \in I\right\}$ of simple roots of $\mathfrak{g}$ with respect to $\mathfrak{h}$ and a set $\left\{\omega_{i}: i \in I\right\}$ of fundamental weights. Let $P$ (resp. $P^{+}$) be the $\mathbb{Z}$ (resp. $\mathbb{Z}_{+}$) span of $\left\{\omega_{i}: i \in I\right\}$. Let $R$ and $R^{+}$be the set of roots and positive roots respectively. For $\alpha \in R^{+}$, let $\mathfrak{s l}_{2}(\alpha)=\left\langle x_{\alpha}^{ \pm}, h_{\alpha}\right\rangle$ be the corresponding subalgebra of $\mathfrak{g}$ isomorphic to $\mathfrak{s l}_{2}$ and set $h_{-\alpha}=-h_{\alpha}$ for $\alpha \in R^{+}$, $h_{i}=h_{\alpha_{i}}$. Let $W$ be the Weyl group of $\mathfrak{g}$ and recall that $W$ acts on $\mathfrak{h}$ and $\mathfrak{h}^{*}$ and that for all $\boldsymbol{w} \in W, \lambda \in \mathfrak{h}^{*}$ and $\alpha \in R^{+}$, we have

$$
(\boldsymbol{w} \lambda)\left(\boldsymbol{w} h_{\alpha}\right)=\lambda\left(h_{\alpha}\right)
$$

For $\alpha \in R^{+}$, let $s_{\alpha} \in W$ be the corresponding reflection and we set $s_{i}=s_{\alpha_{i}}$. Let $\boldsymbol{w}_{0} \in W$ be the longest element. 
We say that $\lambda \in P^{+}$is minuscule if $\lambda\left(h_{\alpha}\right) \in\{0,1\}$ for all $\alpha \in R^{+}$. It can be easily seen that if $\lambda \in P^{+}$is minuscule, then $\lambda$ is a fundamental weight. The following is the list of minuscule weights; here we follow the numbering of vertices of the Dynkin diagram for $\mathfrak{g}$ in [Bourbaki 2002].

$$
\begin{array}{ll}
A_{n} & \omega_{i}, 1 \leq i \leq n \\
B_{n} & \omega_{n} \\
C_{n} & \omega_{1} \\
D_{n} & \omega_{1}, \omega_{n-1}, \omega_{n} \\
E_{6} & \omega_{1}, \omega_{6} \\
E_{7} & \omega_{7}
\end{array}
$$

1.2. For any $\mathfrak{g}$-module $M$ and $\mu \in \mathfrak{h}^{*}$, set

$$
M_{\mu}=\{m \in M: h m=\mu(h) m, h \in \mathfrak{h}\}, \quad \operatorname{wt}(M)=\left\{\mu \in \mathfrak{h}^{*}: M_{\mu} \neq 0\right\} .
$$

We say $M$ is a weight module for $\mathfrak{g}$ if

$$
M=\bigoplus_{\mu \in \mathfrak{h}^{*}} M_{\mu} .
$$

Any finite-dimensional $\mathfrak{g}$-module is a weight module. It is well known that the set of isomorphism classes of irreducible finite-dimensional $\mathfrak{g}$-modules is in bijective correspondence with $P^{+}$. For $\lambda \in P^{+}$we denote by $V(\lambda)$ a representative of the corresponding isomorphism class.

If $V(\lambda)^{*}$ is the dual representation of $V(\lambda)$, then

$$
V(\lambda)^{*} \cong V\left(-\boldsymbol{w}_{0} \lambda\right) .
$$

The dimension of $V(\lambda)$ is given by the Weyl dimension formula, namely

$$
\operatorname{dim} V(\lambda)=\prod_{\alpha \in R^{+}} \frac{(\lambda+\rho)\left(h_{\alpha}\right)}{\rho\left(h_{\alpha}\right)},
$$

where $\rho=\sum_{i=1}^{n} \omega_{i} \in P^{+}$. Any finite-dimensional $\mathfrak{g}$-module is isomorphic to a direct sum of irreducible modules, and in particular, we have

$$
V(\lambda) \otimes V(\mu) \cong \bigoplus_{\nu \in P^{+}} V(v)^{\oplus c_{\lambda, \mu}^{v},} \quad c_{\lambda, \mu}^{\nu}=\operatorname{dim}_{\operatorname{Hom}}(V(\nu), V(\lambda) \otimes V(\mu)) .
$$

We shall freely use the fact that

$$
V(\lambda) \otimes V(\mu) \cong V(\mu) \otimes V(\lambda)
$$


and that

$$
\operatorname{dim} \operatorname{Hom}_{\mathfrak{g}}(V(\nu), V(\lambda) \otimes V(\mu))=\operatorname{dim} \operatorname{Hom}_{\mathfrak{g}}\left(V(\nu)^{*}, V(\lambda)^{*} \otimes V(\mu)^{*}\right) .
$$

\section{The poset $P^{+}(\lambda, k) / \sim$ and the main result}

2.1. Given an integer $k>0$ and $\lambda \in P^{+}$, set

$$
P^{+}(\lambda, k)=\left\{\lambda=\left(\lambda_{1}, \ldots, \lambda_{k}\right) \in\left(P^{+}\right)^{\times k}: \lambda_{1}+\cdots+\lambda_{k}=\lambda\right\} .
$$

Clearly, $P^{+}(\lambda, k)$ is a finite set and the symmetric group $S_{k}$ acts naturally on it. The Weyl group $W$ acts diagonally on $P^{+}(\lambda, k)$ as follows:

$$
w \lambda=w\left(\lambda_{1}, \ldots, \lambda_{k}\right)=\left(w \lambda_{1}, \ldots, w \lambda_{k}\right) \quad \text { for } w \in W .
$$

Observe that

$$
\lambda \in P^{+}(\lambda, k) \Longleftrightarrow-w_{0} \lambda \in P^{+}\left(-w_{0} \lambda, k\right) .
$$

For $\lambda \in P^{+}(\lambda, k), \alpha \in R^{+}$, and $1 \leq \ell \leq k$, set

$$
r_{\alpha, \ell}(\lambda):=\min \left\{\left(\lambda_{i_{1}}+\cdots+\lambda_{i_{\ell}}\right)\left(h_{\alpha}\right): 1 \leq i_{1}<i_{2}<\cdots<i_{\ell} \leq k\right\} .
$$

Clearly, $r_{\alpha, k}(\lambda) \geq r_{\alpha, k-1}(\lambda, \alpha) \geq \cdots \geq r_{\alpha, 1}(\lambda)$. Given $\lambda, \mu \in P^{+}(\lambda, k)$, we say that $\lambda \preceq \mu$ if

$$
r_{\alpha, \ell}(\lambda) \leq r_{\alpha, \ell}(\boldsymbol{\mu}) \quad \text { for all } \alpha \in R^{+} \text {and } 1 \leq \ell \leq k .
$$

This defines a preorder on the $P^{+}(\lambda, k)$. It induces a partial order on the set of equivalence classes with respect to the equivalence relation $\sim$ on $P^{+}(\lambda, k)$, given by

$$
\lambda \sim \boldsymbol{\mu} \Longleftrightarrow r_{\alpha, \ell}(\boldsymbol{\lambda})=r_{\alpha, \ell}(\boldsymbol{\mu}) \quad \text { for all } \alpha \in R^{+} \text {and } 1 \leq \ell \leq k .
$$

The poset has a unique minimal element, which is just the $k$-tuple $(\lambda, 0, \ldots, 0)$.

For ease of notation, we shall not always distinguish between elements of $P^{+}(\lambda, k) / \sim$ and their representatives in $P^{+}(\lambda, k)$.

Note that if $\lambda, \mu \in P^{+}(\lambda, k)$, then

$$
\lambda \preceq \mu \Longleftrightarrow-w_{0} \lambda \preceq-w_{0} \mu .
$$

We now state the main result of this paper.

Theorem 1. Let $\mathfrak{g}$ be a finite-dimensional simple Lie algebra and let $k \in \mathbb{Z}_{+}$and $\lambda \in P^{+}$. Assume that $\lambda=\left(\lambda_{1}, \ldots, \lambda_{k}\right)$ and $\boldsymbol{\mu}=\left(\mu_{1}, \ldots, \mu_{k}\right)$ are elements of $P^{+}(\lambda, k)$ such that $\lambda \preceq \mu$ in $P^{+}(\lambda, k) / \sim$.

(i) We have

$$
\operatorname{dim} \bigotimes_{s=1}^{k} V\left(\lambda_{s}\right) \leq \operatorname{dim} \bigotimes_{s=1}^{k} V\left(\mu_{s}\right),
$$

with equality if and only if $\lambda=\mu$ in $P^{+}(\lambda, k) / \sim$. 
(ii) Let $i \in I$ be such that $\omega_{i}$ is minuscule and let $\lambda=N \omega_{i}$ for some $N \in \mathbb{Z}_{+}$. Then $\operatorname{dim} \operatorname{Hom}_{\mathfrak{g}}\left(V(v), \bigotimes_{s=1}^{k} V\left(\lambda_{s}\right)\right) \leq \operatorname{dim} \operatorname{Hom}_{\mathfrak{g}}\left(V(v), \bigotimes_{s=1}^{k} V\left(\mu_{s}\right)\right), \quad v \in P^{+}$.

(iii) If $\mathfrak{g}$ is of type $A_{2}$, and $k=2$, then

$\lambda \preceq \boldsymbol{\mu} \Longrightarrow \operatorname{dim} \operatorname{Hom}_{\mathfrak{g}}\left(V(v), V\left(\lambda_{1}\right) \otimes V\left(\lambda_{2}\right)\right) \leq \operatorname{dim}_{\operatorname{Hom}}\left(V(v), V\left(\mu_{1}\right) \otimes V\left(\mu_{2}\right)\right)$, for all $v \in P^{+}$.

The theorem is proved in the rest of this paper. We conclude this section with some comments on the methods of the proof and give some explanations for the restrictions in the theorem. We also give some context to our result by relating it to others in the literature.

2.2. To prove part (i) of the theorem, we use the Weyl dimension formula to reduce the proof to the case of $\mathfrak{s l}_{2}$. Recall that the cover relation of $\preceq$ on $P^{+}(\lambda, k)$ is the pairs $\lambda \prec \boldsymbol{\mu}$, such that there does not exist $\boldsymbol{v} \in P^{+}(\lambda, k)$ with $\lambda \prec \boldsymbol{v} \prec \boldsymbol{\mu}$. For $\mathfrak{s l}_{2}$ we determine this cover relation, which allows us to determine a sufficient condition for the cover relation in $P^{+}(\lambda, k)$ in general. Part (ii) of the theorem follows from these ideas along with the Littelmann path model. For an arbitrary simple Lie algebra, it seems quite difficult to determine the cover relations even for $k=2$. For $\mathfrak{s l}_{3}$ and $k=2$, we give a sufficient condition for one element to cover another in Section 5. It would appear from our conditions that the cover relation depends heavily on the combinatorics of the Weyl group and the root system. Part (iii) of the theorem is proved by using the information on the cover relation together with results of [Kashiwara and Nakashima 1994; Nakashima 1993] on realization of crystal bases.

2.3. A subject that has been of much interest has been the notion of Schur positivity. Let $\Lambda$ be the ring of symmetric functions. It is well known [Macdonald 1995] that it has an integral basis given by the Schur functions $s_{\chi}$, where $\chi$ is a partition. A symmetric function is said to be Schur positive if it can be written as a nonnegative integer linear combination of Schur functions.

Suppose now that $\mathfrak{g}$ is of type $A_{r-1}$ and $\lambda \in P^{+}$, say $\lambda=\sum_{i=1}^{r-1} s_{i} \omega_{i}$. Define a partition $\chi(\lambda)=\left(\chi_{1} \geq \cdots \geq \chi_{r}\right)$ by $\chi_{j}=\sum_{p=j}^{r-1} s_{p}$ if $1 \leq j \leq r-1$ and set $\chi_{r}=0$. On the other hand, given a partition $\chi=\left(\chi_{1} \geq \chi_{2} \geq \cdots \geq \chi_{r-1} \geq 0\right)$, set $\lambda(\chi)=\sum_{i=1}^{r-1}\left(\chi_{i}-\chi_{i+1}\right) \omega_{i} \in P^{+}$.

It is known that the character of $V(\lambda)$ is $s_{\chi}(\lambda)$. Parts (ii) and (iii) of Theorem 1 can be reformulated as: let $\lambda=\left(\lambda_{1}, \lambda_{2}\right), \boldsymbol{\mu}=\left(\mu_{1}, \mu_{2}\right) \in P^{+}(\lambda, 2)$; then

$$
\lambda \preceq \boldsymbol{\mu} \Longrightarrow s_{\chi\left(\mu_{1}\right)} s_{\chi\left(\mu_{2}\right)}-s_{\chi\left(\lambda_{1}\right)} s_{\chi\left(\lambda_{2}\right)}=\sum_{\nu \in P^{+}} c_{\nu} s_{\chi(\nu)}, \quad c_{\nu} \geq 0 \text { for all } v \in P^{+} .
$$

We conjecture that this is true more generally: 
Conjecture. Let $\mathfrak{g}$ be a simple Lie algebra and let $\lambda \in P^{+}$and $\lambda=\left(\lambda_{1}, \lambda_{2}\right)$, $\boldsymbol{\mu}=\left(\mu_{1}, \mu_{2}\right) \in P^{+}(\lambda, 2)$; then

$\lambda \preceq \boldsymbol{\mu} \Longrightarrow \operatorname{dim} \operatorname{Hom}_{\mathfrak{g}}\left(V(v), V\left(\lambda_{1}\right) \otimes V\left(\lambda_{2}\right)\right) \leq \operatorname{dim}_{\operatorname{Hom}}\left(V(v), V\left(\mu_{1}\right) \otimes V\left(\mu_{2}\right)\right)$,

for all $v \in P^{+}$.

Part (i) of the theorem could be viewed as giving some additional, but very limited evidence for the conjecture.

2.4. In [Fomin et al. 2005] the authors introduced the notion of a row shuffle. Thus if $\chi=\left(\chi_{1} \geq \chi_{2} \geq \cdots \geq \chi_{n-1} \geq 0\right)$ and $\xi=\left(\xi_{1} \geq \xi_{2} \geq \cdots \geq\right.$ $\left.\xi_{n-1} \geq 0\right)$ are two partitions with at most $n-1$ parts, then the row shuffle is a pair of partitions $\left(\rho^{1}(\chi, \xi), \rho^{2}(\chi, \xi)\right)$ defined as follows. Order the multiset $\left\{\chi_{1}, \ldots, \chi_{r-1}, \xi_{1}, \ldots, \xi_{n-1}\right\}$ decreasingly, say $\psi_{1} \geq \psi_{2} \geq \cdots \geq \psi_{2 n-2}$. Set

$$
\begin{aligned}
& \rho^{1}(\chi, \xi)=\left(\psi_{1} \geq \psi_{3} \geq \cdots \geq \psi_{2 n-3} \geq 0\right), \\
& \rho^{2}(\chi, \xi)=\left(\psi_{2} \geq \psi_{4} \geq \cdots \geq \psi_{2 n-2} \geq 0\right) .
\end{aligned}
$$

In other words, we are shuffling the rows of the joint partition. It was conjectured in [Fomin et al. 2005] and proved in [Lam et al. 2007] that $s_{\rho^{1}(\chi, \xi)} s_{\rho^{2}(\chi, \xi)}-s_{\chi} s \xi$ is Schur positive. Related conjectures can also be found in [Okounkov 1997; Lascoux et al. 1997]. Partial results on this conjecture were also obtained in [Bergeron et al. 2006; Fran and McNamara 2004; McNamara 2008; McNamara and van Willigenburg 2009; Purbhoo and van Willigenburg 2008].

2.5. The connection of the row shuffle with our partial order is made as follows. Suppose that $\mathfrak{g}$ is of type $A_{n-1}$, let $v, \mu \in P^{+}$, and set $\lambda=\mu+v$. In Section 5.3, we define an element $\lambda_{\max }=\left(\lambda^{1}, \lambda^{2}\right) \in P^{+}(\lambda, 2) / \sim$ and prove that it is the unique maximal element in this set. It is a simple calculation to prove that if we take $\xi$ and $\chi$ to be the duals of the partitions $\chi(v)$ and $\chi(\mu)$, then $\rho^{1}(\xi, \chi)$ and $\rho^{2}(\xi, \chi)$ are the duals of the partitions $\chi\left(\lambda^{1}\right)$ and $\chi\left(\lambda^{2}\right)$, respectively. It is well known [Macdonald 1995] that there exists an involution $\omega$ of the ring $\Lambda$ which maps the Schur function associated to a partition $\xi$ to the Schur function associated to its dual. Hence the result of [Lam et al. 2007] proves the following statement: let $\mu, v \in P^{+}$and let $\lambda=\mu+v$. Then

$\operatorname{dim} \operatorname{Hom}_{\mathfrak{g}}(V(\eta), V(\mu) \otimes V(\nu)) \leq \operatorname{dim} \operatorname{Hom}_{\mathfrak{g}}\left(V(\eta), V\left(\lambda^{1}\right) \otimes V\left(\lambda^{2}\right)\right)$ for all $\eta \in P^{+}$.

In other words, their result proves that part (iii) of Theorem 1 is true for $A_{n-1}$ in the case when $\boldsymbol{\mu}=\lambda_{\max }$.

We remark that in [Lam et al. 2007] the authors proved, further, that in the case where $\mathfrak{g}$ is of type $A_{n-1}$, having Schur positivity for $k=2$ (and $\boldsymbol{\mu}=\lambda_{\max }$ ) implies 
Schur positivity for arbitrary $k$; for example, they proved $\operatorname{dim} \operatorname{Hom}_{\mathfrak{g}}\left(V(\eta), V\left(\mu_{1}\right) \otimes \cdots \otimes V\left(\mu_{k}\right)\right) \leq \operatorname{dim} \operatorname{Hom}_{\mathfrak{g}}\left(V(\eta), V\left(\lambda^{1}\right) \otimes \cdots \otimes V\left(\lambda^{k}\right)\right)$ for all $k \geq 2,\left(\mu_{1}, \ldots, \mu_{k}\right) \in \mathscr{P}(\lambda, k)$ and $\eta \in P^{+}$, where $\left(\lambda^{1}, \ldots, \lambda^{k}\right)$ is the $k$-row shuffle of $\left(\mu_{1}, \ldots, \mu_{k}\right)$. One can show (similar to the proposition in 5.3) that $\left(\lambda^{1}, \ldots, \lambda^{k}\right)$ is the unique maximal element in $\mathscr{P}(\lambda, k)$.

2.6. Suppose $\mathfrak{g}$ is of type $A_{n}$; then Lam, Postnikov and Pylyavskyy stated the conjecture in 2.3 in an unpublished work (we may refer here to [Dobrovolska and Pylyavskyy 2007]). The following first step in proving this conjecture in the $A_{n}$-case has been taken in [Dobrovolska and Pylyavskyy 2007]. It is shown there that for $\left(\mu_{1}, \mu_{2}\right) \preceq\left(\lambda_{1}, \lambda_{2}\right) \in P^{+}(\lambda, 2)$ and $v \in P^{+}$,

$\operatorname{dim} \operatorname{Hom}_{\mathfrak{g}}\left(V(v), V\left(\mu_{1}\right) \otimes V\left(\mu_{2}\right)\right) \neq 0 \Longrightarrow \operatorname{dim} \operatorname{Hom}_{\mathfrak{g}}\left(V(v), V\left(\lambda_{1}\right) \otimes V\left(\lambda_{2}\right)\right) \neq 0$.

Their approach is completely different from ours, as they use the Horn-Klyachko inequalities. It would be interesting to see if some of the ideas apply also when $\mathfrak{g}$ is not of type $A_{n}$.

\section{Proof of Theorem 1(i)}

The main idea in the proof of part (i) of the theorem is to show that when $\lambda$ is a multiple of a fundamental weight, the partial order on $P^{+}(\lambda, k)$ is determined by the partial order on $P^{+}(\lambda, 2)$. We prove this in the first part of the section and then deduce Theorem 1(i).

3.1. Assume until further notice that $\mathfrak{g}$ is an arbitrary finite-dimensional simple Lie algebra, and

$$
\lambda \in \mathbb{Z}_{+} \omega_{i} \text { for some } i \in I .
$$

In particular, we can and will think of $\lambda$ as a nonnegative integer. It is clear that elements of $P^{+}(\lambda, k)$ are just $k$-tuples of nonnegative integers which add up to $\lambda \in \mathbb{Z}_{+}$. If $\lambda \in P^{+}(\lambda, k)$, we have

$$
r_{\alpha, \ell}(\lambda)=d_{i} r_{\alpha_{i}, \ell}(\lambda), \quad h_{\alpha}=\sum_{i \in I} d_{i} h_{i},
$$

and it follows that if $\lambda, \mu \in P^{+}(\lambda, k)$, then

$$
\lambda \preceq \boldsymbol{\mu} \Longleftrightarrow r_{\alpha_{i}, \ell}(\lambda) \leq r_{\alpha_{i}, \ell}(\boldsymbol{\mu}), \quad 1 \leq \ell \leq k .
$$

In other words, the partial order is determined entirely by $\alpha_{i}$. So we shall drop the dependence on $\alpha$ and write $r_{\ell}(\lambda)$ for $r_{\alpha_{i}, \ell}(\lambda)$. 
3.2.

Lemma. Let $\lambda, \boldsymbol{\mu} \in P^{+}(\lambda, k)$. Then $\lambda \sim \boldsymbol{\mu}$ if and only if $\boldsymbol{\mu}=\sigma \lambda$ for some $\sigma \in S_{k}$, that is, the equivalence class of $\lambda$ is exactly the $S_{k}$ orbit of $\lambda$.

Proof. The fact that $\lambda=\sigma \boldsymbol{\mu}$ implies $\lambda \sim \boldsymbol{\mu}$ is clear from the definition of $P^{+}(\lambda, k)$. For the converse, choose $\sigma, \sigma^{\prime} \in S_{k}$ so that $\sigma \lambda$ and $\sigma^{\prime} \mu$ are partitions of $\lambda$, say

$$
\sigma \lambda=\left(\lambda_{k} \geq \cdots \geq \lambda_{1}\right), \quad \sigma^{\prime} \mu=\left(\mu_{k} \geq \cdots \geq \mu_{1}\right) .
$$

Since $\lambda \sim \sigma \lambda$, it follows that $\sigma \lambda \sim \sigma^{\prime} \boldsymbol{\mu}$. But this implies that

$$
\lambda_{1}=\mu_{1}, \quad \lambda_{1}+\lambda_{2}=\mu_{1}+\mu_{2}, \quad \ldots, \quad \lambda_{1}+\lambda_{2}+\cdots+\lambda_{k}=\mu_{1}+\cdots+\mu_{k},
$$

forcing $\sigma \lambda=\sigma^{\prime} \boldsymbol{\mu}$, and the Lemma is proved.

From now on, we will identify the set $P^{+}(\lambda, k) / \sim$ of equivalence classes with partitions of $\lambda$ with at most $k$ parts. By abuse of notation, we continue to denote this set by $P^{+}(\lambda, k)$; note that $\preceq$ is now a partial order on this set. As a consequence, we shall also assume without comment that $r_{\ell}(\lambda)=\lambda_{1}+\cdots+\lambda_{\ell}$ for $1 \leq \ell \leq k$.

3.3. Let $\lambda$ be as in (3-1), and let $k \in \mathbb{Z}_{+}$. Write $\lambda=k \lambda_{0}+p_{0}$, where $0 \leq p_{0}<k$ and $\lambda_{0} \in \mathbb{Z}_{+}$. Define $\lambda_{\max }=\left(\lambda_{k} \geq \cdots \geq \lambda_{1}\right) \in P^{+}(\lambda, k)$ by

$$
\lambda_{j}= \begin{cases}\lambda_{0} & 1 \leq j \leq k-p_{0} \\ \lambda_{0}+1 & j>k-p_{0}\end{cases}
$$

Observe that

$$
r_{\ell}\left(\lambda_{\max }\right)= \begin{cases}\ell \lambda_{0} & \text { if } 1 \leq \ell \leq k-p_{0} \\ (\ell+1) \lambda_{0}-k+p_{0} & \text { otherwise }\end{cases}
$$

The following result justifies the notation.

Lemma. Keep the notation above. For all $\lambda \in P^{+}(\lambda, k)$, we have $\lambda \preceq \lambda_{\max }$. Moreover, $\lambda_{\max }$ is the unique element of $P^{+}(\lambda, k)$ satisfying

$$
\max _{1 \leq i \leq k}\left\{\lambda_{i}\right\}-\min _{1 \leq i \leq k}\left\{\lambda_{i}\right\} \leq 1 .
$$

Proof. It is clear that $\lambda_{\max }$ satisfies (3-2). If $\boldsymbol{\mu}=\left(\mu_{k} \geq \cdots \geq \mu_{1}\right) \in P^{+}(\lambda, k)$ also satisfies (3-2), then $\mu_{j}-\mu_{1} \leq 1$ for all $1 \leq j \leq k$. If $\mu_{j}=\mu_{1}$ for all $j$, then $\lambda=k \mu_{1}$, and hence we would have $p_{0}=0$ and $\mu_{1}=\lambda_{0}$ as required. Otherwise, fix $j_{0} \leq k-1$ such that $\mu_{j}=\mu_{1}$ for $1 \leq j \leq k-j_{0}$ and $\mu_{j}=\mu_{1}+1$ otherwise. Then

$$
\sum_{s=1}^{k} \mu_{s}=k \mu_{1}+j_{0}=\lambda=k \lambda_{0}+k_{0}=\sum_{s=1}^{k} \lambda_{s},
$$

and hence $\mu_{1}=\lambda_{0}$ and $j_{0}=k_{0}$, which proves that $\boldsymbol{\mu}=\lambda_{\max }$. 
Suppose for a contradiction that there exist $\lambda=\left(\lambda_{k} \geq \cdots \geq \lambda_{1}\right) \in P^{+}(\lambda, k)$ and $1 \leq j \leq k-p_{0}$ with $r_{j}(\lambda)>j \lambda_{0}$, and assume that $j$ is minimal with this property. Since

$$
r_{j}(\lambda)=\lambda_{j}+r_{j-1}(\lambda)>j \lambda_{0},
$$

we get $\lambda_{\ell} \geq \lambda_{j} \geq \lambda_{0}+1$ for all $\ell \geq j$. This gives

$$
\lambda=\lambda_{k}+\cdots+\lambda_{j+1}+r_{j}(\lambda)>(k-j)\left(\lambda_{0}+1\right)+j \lambda_{0} \geq k \lambda_{0}+p_{0}=\lambda,
$$

which is a contradiction. The case $j>k-p_{0}$ is similar, and we omit the details.

3.4. We now prove that the partial order on $P^{+}(\lambda, k)$ is entirely determined by the partial order $P^{+}(\lambda, 2)$. The first step is the following result, which determines the cover relation in $P^{+}(\lambda, 2)$.

Lemma. Suppose that $\lambda=\left(\lambda_{1} \geq \lambda_{2}\right) \in P^{+}(\lambda, 2)$ and assume that $\lambda \neq \lambda_{\max }$. Then $\boldsymbol{\mu} \in P^{+}(\lambda, 2)$ covers $\lambda$ if and only if

$$
\boldsymbol{\mu}=\left(\lambda_{2}-1 \geq \lambda_{1}+1\right) .
$$

Proof. Since $\lambda \neq \lambda_{\max }$, we see from the lemma in 3.3 that $\lambda_{2}-\lambda_{1}>1$. Hence, $\boldsymbol{\mu}=\left(\lambda_{2}-1 \geq \lambda_{1}+1\right) \in P^{+}(\lambda, k)$ and $\boldsymbol{\lambda} \preceq \boldsymbol{\mu}$. Suppose that there exists $\boldsymbol{v}=\left(v_{2} \geq v_{1}\right)$ with $\lambda \prec \boldsymbol{v}$. Then $\lambda_{1}<v_{1}$, and hence we get $\boldsymbol{\mu} \preceq \boldsymbol{v}$, which proves the lemma.

3.5. Let $\lambda=\left(\lambda_{k} \geq \cdots \geq \lambda_{1}\right) \in P^{+}(\lambda, k)$ be such that $\lambda_{k}-\lambda_{1} \geq 2$. Then there exist $1 \leq j_{1}<j_{2} \leq k$ with

$$
\lambda_{j_{1}}<\lambda_{j_{1}+1}, \quad \lambda_{j_{2}-1}<\lambda_{j_{2}}, \quad \lambda_{j_{1}}+2 \leq \lambda_{j_{2}} .
$$

For each such pair $\left(j_{2}, j_{1}\right)$, we define a partition $\lambda\left(j_{2}, j_{1}\right)=\left(\lambda_{k}^{\prime} \geq \cdots \geq \lambda_{1}^{\prime}\right)$ :

$$
\lambda_{i}^{\prime}= \begin{cases}\lambda_{i} & i \neq j_{1}, j_{2}, \\ \lambda_{j_{2}}-1 & i=j_{2}, \\ \lambda_{j_{1}}+1 & i=j_{1} .\end{cases}
$$

Observe that

$$
r_{\ell}\left(\lambda\left(j_{2}, j_{1}\right)\right)= \begin{cases}r_{\ell}(\lambda)+1 & j_{1} \leq \ell<j_{2} \\ r_{\ell}(\lambda) & \text { otherwise }\end{cases}
$$

and so $\lambda \prec \lambda\left(j_{2}, j_{1}\right)$. The following proposition shows that the partial order on $P^{+}(\lambda, k)$ is controlled by the partial order on $P^{+}(\lambda, 2)$.

Proposition. Let $\lambda=\left(\lambda_{k} \geq \cdots \geq \lambda_{1}\right) \in P^{+}(\lambda, k)$ and assume that $\mu \in P^{+}(\lambda, k)$ covers $\lambda$. Then $\boldsymbol{\mu}=\lambda\left(j_{2}, j_{1}\right)$ for some $1 \leq j_{1}<j_{2} \leq k$ with $\lambda_{j_{1}}<\lambda_{j_{1}+1}$ and $\lambda_{j_{2}-1}<\lambda_{j_{2}}$ and $\lambda_{j_{1}}+2 \leq \lambda_{j_{2}}$. 
Proof. We proceed by induction on $k$. The lemma in 3.4 shows that induction begins at $k=2$. Assume that $k>2$. Let $\lambda \in P^{+}(\lambda, k)$, and assume that $\mu \in P^{+}(\lambda, k)$ covers $\lambda$.

Suppose $r_{\ell}(\lambda)<r_{\ell}(\boldsymbol{\mu})$ for all $1 \leq \ell<k$. Since $\lambda$ has a cover, it follows that $\lambda \neq \lambda_{\max }$, and so there exist $1 \leq j_{1}<j_{2} \leq \ell$ such that $\lambda\left(j_{2}, j_{1}\right)$ is defined. Now, (3-4) shows that

$$
\lambda \prec \lambda\left(j_{2}, j_{1}\right) \preceq \mu,
$$

and hence $\boldsymbol{\mu}=\lambda\left(j_{2}, j_{1}\right)$.

Suppose now that there exists $1 \leq \ell<k$ such that $r_{\ell}(\boldsymbol{\mu})=r_{\ell}(\lambda)$ and $\ell$ is minimal with this property. Consider first the case when $\ell=1$, that is, $\mu_{1}=\lambda_{1}$. Then

$$
\mu_{0}=\left(\mu_{k} \geq \cdots \geq \mu_{2}\right), \quad \lambda_{0}=\left(\lambda_{k} \geq \cdots \geq \lambda_{2}\right)
$$

are distinct elements of $P^{+}\left(\lambda-\lambda_{1}, k-1\right)$ (since $\left.\mu \neq \lambda\right)$. Moreover, we claim that $\boldsymbol{\mu}_{0}$ covers $\lambda_{0}$. If there exists $\boldsymbol{v}_{0}=\left(v_{k} \geq \cdots \geq v_{2}\right) \in P^{+}\left(\lambda-\lambda_{1}, k-1\right)$ such that

$$
\lambda_{0} \prec v_{0} \preceq \mu_{0},
$$

then $v_{2} \geq \lambda_{2} \geq \lambda_{1}$. Hence, if we set $\boldsymbol{v}=\left(v_{k} \geq \cdots \geq v_{2} \geq \lambda_{1}\right) \in P^{+}(\lambda, k)$, then we get

$$
\lambda \prec v \preceq \mu .
$$

This forces $\boldsymbol{v}=\boldsymbol{\mu}$, and hence $\boldsymbol{v}_{0}=\boldsymbol{\mu}_{0}$. By induction on $k$ and noting that $k-1 \geq 2$, we see that

$$
\boldsymbol{\mu}_{0}=\lambda_{0}\left(j_{2}, j_{1}\right)
$$

for some $2 \leq j_{1}<j_{2} \leq k$, and hence $\boldsymbol{\mu}=\lambda\left(j_{2}, j_{1}\right)$.

It remains to consider the case when $\ell \geq 2$, which in particular would imply that $\mu_{1}>\lambda_{1}$. This time, we take

$$
\mu_{0}=\left(\mu_{\ell} \geq \cdots \geq \mu_{1}\right), \quad \lambda_{0}=\left(\lambda_{\ell} \geq \cdots \geq \lambda_{1}\right)
$$

and note that these are elements of $P^{+}\left(r_{\ell}(\lambda), \ell\right)$ and that $\lambda_{0} \prec \mu_{0}$. We claim again that $\mu_{0}$ covers $\lambda_{0}$. Thus, let $\boldsymbol{v}_{0}=\left(v_{\ell} \geq \cdots \geq v_{1}\right) \in P^{+}\left(r_{\ell}(\lambda), \ell\right)$ be such that

$$
\lambda_{0} \prec v_{0} \preceq \mu_{0} .
$$

Then

$$
v_{s}+\cdots+v_{1} \geq \lambda_{s}+\cdots+\lambda_{1}, \quad 1 \leq s \leq \ell, \quad v_{\ell}+\cdots+v_{1}=\lambda_{\ell}+\cdots+\lambda_{1} .
$$

Suppose that $v_{\ell}>\lambda_{\ell+1}$. Then we get $v_{\ell}>\lambda_{\ell+1} \geq \lambda_{\ell}$ and

$$
0<v_{\ell}-\lambda_{\ell}=\left(\lambda_{\ell-1}+\cdots+\lambda_{1}\right)-\left(v_{\ell-1}+\cdots+v_{1}\right) \leq 0,
$$

which is a contradiction. Thus we get $v_{\ell} \leq \lambda_{\ell+1}$, and hence

$$
\boldsymbol{v}=\left(\lambda_{k} \geq \cdots \geq \lambda_{\ell+1} \geq v_{\ell} \geq \cdots \geq v_{1}\right) \in P^{+}(\lambda, k) .
$$


Also we see that $\boldsymbol{\lambda} \prec \boldsymbol{v} \preceq \boldsymbol{\mu}$. Since $\boldsymbol{\mu}$ is a cover of $\boldsymbol{\lambda}$, this forces $\boldsymbol{v}=\boldsymbol{\mu}$, and hence $\boldsymbol{v}_{0}=\boldsymbol{\mu}_{0}$. Thus we conclude that $\boldsymbol{\mu}_{0}$ covers $\lambda_{0}$. By induction on $k, \boldsymbol{\mu}_{0}=\lambda_{0}\left(j_{2}, j_{1}\right)$ for some $1 \leq j_{1}<j_{2} \leq \ell$. We see that $\lambda \prec \lambda\left(j_{2}, j_{1}\right) \preceq \boldsymbol{\mu}$, which forces $\boldsymbol{\mu}=\lambda\left(j_{2}, j_{1}\right)$. Thus we have proved the proposition.

3.6. Proof of Theorem 1(i). Assume first that $\mathfrak{g}$ is $\mathfrak{s l}_{2}$. Let $\lambda \in P^{+}=\mathbb{Z}_{+} \omega_{1}$ be an arbitrary dominant integral weight, and let $k \geq 2$. Let $\lambda=\left(\lambda_{k} \geq \cdots \geq \lambda_{1}\right) \in P^{+}(\lambda, k)$ and $\boldsymbol{\mu}=\left(\mu_{k} \geq \cdots \geq \mu_{1}\right) \in P^{+}(\lambda, k)$ be such that $\lambda \preceq \boldsymbol{\mu}$ (in $\left.P^{+}(\lambda, k) / \sim\right)$. First we show that if $\lambda \prec \boldsymbol{\mu}$ (in $\left.P^{+}(\lambda, k) / \sim\right)$, then

$$
\operatorname{dim} \bigotimes_{s=1}^{k} V\left(\lambda_{s}\right)<\operatorname{dim} \bigotimes_{s=1}^{k} V\left(\mu_{s}\right), \quad \text { that is, } \prod_{s=1}^{k}\left(\lambda_{s}+1\right)<\prod_{s=1}^{k}\left(\mu_{s}+1\right) .
$$

A standard argument shows that there exists a sequence $\lambda=\boldsymbol{v}_{0}, \boldsymbol{v}_{1}, \ldots, \boldsymbol{v}_{p}=\boldsymbol{\mu}$ of elements of $P^{+}(\lambda, k)$ such that $\boldsymbol{v}_{q}$ covers $\boldsymbol{v}_{q-1}$ for each $1 \leq q \leq p$. It suffices to show (3-5) in the case when $\boldsymbol{\mu}$ covers $\lambda$. Then, by the proposition in 3.5, there exist $1 \leq j_{1}<j_{2} \leq k$ with $\lambda_{j_{1}}<\lambda_{j_{1}+1}$ and $\lambda_{j_{2}-1}<\lambda_{j_{2}}$ and $\lambda_{j_{1}}+2 \leq \lambda_{j_{2}}$ such that $\boldsymbol{\mu}=\lambda\left(j_{2}, j_{1}\right)$. Thus the inequality (3-5) is equivalent to

$$
\left(\lambda_{j_{1}}+1\right)\left(\lambda_{j_{2}}+1\right)<\left(\lambda_{j_{1}}+2\right) \lambda_{j_{2}} .
$$

But this is obvious from the fact that $\lambda_{j_{1}}+2 \leq \lambda_{j_{2}}$. Thus we have proved (3-5). Also, we have proved (under the assumption that $\lambda \preceq \boldsymbol{\mu}$ ) that if

$$
\operatorname{dim} \bigotimes_{s=1}^{k} V\left(\lambda_{s}\right)=\operatorname{dim} \bigotimes_{s=1}^{k} V\left(\mu_{s}\right)
$$

then $\lambda=\mu$ (in $P^{+}(\lambda, k) / \sim$ ). The converse of this statement is obvious by the lemma in 3.2. Thus we have proved Theorem 1(i) in the case of $\mathfrak{s l}_{2}$.

Assume next that $\mathfrak{g}$ is an arbitrary finite-dimensional simple complex Lie algebra, and that $\lambda \in P^{+}$is an arbitrary dominant integral weight. Let $\lambda=\left(\lambda_{1}, \ldots, \lambda_{k}\right)$, $\boldsymbol{\mu}=\left(\mu_{1}, \ldots, \mu_{k}\right) \in P^{+}(\lambda, k)$ be such that $\lambda \preceq \boldsymbol{\mu}$ (in $P^{+}(\lambda, k) / \sim$ ). Using the Weyl dimension formula, we see that

$$
\begin{aligned}
& \operatorname{dim} \bigotimes_{s=1}^{k} V\left(\lambda_{s}\right)=\prod_{s=1}^{k} \prod_{\alpha \in R^{+}} \frac{\left(\lambda_{s}+\rho\right)\left(h_{\alpha}\right)}{\rho\left(h_{\alpha}\right)}, \\
& \operatorname{dim} \bigotimes_{s=1}^{k} V\left(\mu_{s}\right)=\prod_{s=1}^{k} \prod_{\alpha \in R^{+}} \frac{\left(\mu_{s}+\rho\right)\left(h_{\alpha}\right)}{\rho\left(h_{\alpha}\right)} .
\end{aligned}
$$

So, in order to prove that $\operatorname{dim} \bigotimes_{s=1}^{k} V\left(\lambda_{s}\right) \leq \operatorname{dim} \bigotimes_{s=1}^{k} V\left(\mu_{s}\right)$, it suffices to show that 


$$
\prod_{s=1}^{k}\left(\lambda_{s}+\rho\right)\left(h_{\alpha}\right) \leq \prod_{s=1}^{k}\left(\mu_{s}+\rho\right)\left(h_{\alpha}\right) \quad \text { for each } \alpha \in R^{+} .
$$

For each $\alpha \in R^{+}$and $1 \leq s \leq k$, we set

$$
\begin{aligned}
\lambda_{s}^{(\alpha)} & =\left(\lambda_{s}+\rho\right)\left(h_{\alpha}\right)-1, \\
\mu_{s}^{(\alpha)} & =\left(\mu_{s}+\rho\right)\left(h_{\alpha}\right)-1, \\
\lambda^{(\alpha)} & =\lambda\left(h_{\alpha}\right)+k\left(\rho\left(h_{\alpha}\right)-1\right)=\sum_{s=1}^{k} \lambda_{s}^{(\alpha)}=\sum_{s=1}^{k} \mu_{s}^{(\alpha)} .
\end{aligned}
$$

Then we see that the elements

$$
\lambda^{(\alpha)}=\left(\lambda_{1}^{(\alpha)}, \ldots, \lambda_{k}^{(\alpha)}\right), \quad \mu^{(\alpha)}=\left(\mu_{1}^{(\alpha)}, \ldots, \mu_{k}^{(\alpha)}\right)
$$

are elements of $P^{+}\left(\lambda^{(\alpha)}, k\right)$ for $\mathfrak{s l}_{2}$ (or rather $\mathfrak{s l}_{2}(\alpha)$ ) satisfying $\lambda^{(\alpha)} \preceq \boldsymbol{\mu}^{(\alpha)}$ (in $\left.P^{+}\left(\lambda^{(\alpha)}, k\right) / \sim\right)$. Hence, by the argument for $\mathfrak{s l}_{2}$ above, we obtain

$$
\prod_{s=1}^{k}\left(\lambda_{s}+\rho\right)\left(h_{\alpha}\right)=\prod_{s=1}^{k}\left(\lambda_{s}^{(\alpha)}+1\right) \leq \prod_{s=1}^{k}\left(\mu_{s}^{(\alpha)}+1\right)=\prod_{s=1}^{k}\left(\mu_{s}+\rho\right)\left(h_{\alpha}\right),
$$

as desired. Also, we deduce that (under the assumption that $\lambda \preceq \boldsymbol{\mu}$ )

$$
\begin{aligned}
\operatorname{dim} \bigotimes_{s=1}^{k} V\left(\lambda_{s}\right) & =\operatorname{dim} \bigotimes_{s=1}^{k} V\left(\mu_{s}\right) \\
& \Longleftrightarrow \prod_{s=1}^{k}\left(\lambda_{s}^{(\alpha)}+1\right)=\prod_{s=1}^{k}\left(\mu_{s}^{(\alpha)}+1\right) \quad \text { for all } \alpha \in R^{+} \\
& \Longleftrightarrow \lambda^{(\alpha)}=\mu^{(\alpha)} \quad \text { for all } \alpha \in R^{+} \text {(by the argument for } \mathfrak{s l}_{2} \text { above) } \\
& \Longleftrightarrow \lambda=\mu .
\end{aligned}
$$

Thus we have proved Theorem 1(i).

\section{Proof of Theorem 1(ii)}

4.1. As in Section 3, we regard elements of $P^{+}\left(N \omega_{i}, k\right)$ as partitions of $N$. Also, we deduce from the proposition in 3.5 that Theorem 1(ii) is proved once we establish the following proposition.

Proposition. Suppose that $r, s \in \mathbb{Z}_{+}$and assume that $s \geq r+1$. Let $i \in I$ be such that $\omega_{i}$ is minuscule. Then, for all $\mu \in P^{+}$, we have

$\operatorname{dim} \operatorname{Hom}_{\mathfrak{g}}\left(V(\mu), V\left(s \omega_{i}\right) \otimes V\left(r \omega_{i}\right)\right)$

$$
\leq \operatorname{dim} \operatorname{Hom}_{\mathfrak{g}}\left(V(\mu), V\left((s-1) \omega_{i}\right) \otimes V\left((r+1) \omega_{i}\right)\right) .
$$


The proposition is established in the rest of the section using the Littelmann path model.

4.2. We recall the essential definitions and results from [Littelmann 1994; 1995].

Definition. (i) Let $\lambda \in P^{+}$and $\mu, v \in W \lambda$. We say that $\mu \geq v$ if there exists a sequence $\mu=\xi_{0}, \xi_{1}, \ldots, \xi_{m}=v$ of elements in $W \lambda$ and elements $\beta_{1}, \ldots, \beta_{m} \in$ $R^{+}$of positive roots such that

$$
\xi_{p}=s_{\beta_{p}}\left(\xi_{p-1}\right), \quad \xi_{p-1}\left(h_{\beta_{p}}\right)<0, \quad 1 \leq p \leq m .
$$

Moreover, in this case, we let $\operatorname{dist}(\mu, v)$ be the maximal length $m$ of all such possible sequences.

(ii) For $\mu, v \in W \lambda$ with $\mu>v$ and a rational number $0<a<1$, we define an $a$-chain for $(\mu, v)$ as a sequence $\mu=\xi_{0}>\xi_{1}>\cdots>\xi_{m}=v$ of elements in $W \lambda$ such that

$$
\operatorname{dist}\left(\xi_{p-1}, \xi_{p}\right)=1, \quad \xi_{p}=s_{\beta_{p}}\left(\xi_{p-1}\right), \quad a \xi_{p-1}\left(h_{\beta_{p}}\right) \in \mathbb{Z}_{<0}
$$

for all $p=1,2, \ldots, m$.

(iii) An LS path of shape $\lambda$ is a pair $(\underline{v} ; \underline{a})$ consisting of a sequence

$$
\underline{v}=\left(v_{1}>v_{2}>\cdots>v_{\ell}\right) \quad(\text { for some } \ell \geq 1)
$$

of elements in $W \lambda$ and a sequence $\underline{a}=\left(0=a_{0}<a_{1}<\cdots<a_{\ell}=1\right)$ of rational numbers satisfying the condition that there exists an $a_{p}$-chain for $\left(v_{p}, v_{p+1}\right)$ for $p=1,2, \ldots, \ell-1$. We denote by $\mathbb{B}(\lambda)$ the set of all LS paths of shape $\lambda$.

4.3. Set $\mathfrak{h}_{\mathbb{R}}^{*}=\sum_{i=1}^{n} \mathbb{R} \omega_{i}$, where $\mathbb{R}$ is the set of real numbers. Given an LS path $\pi=(\underline{v} ; \underline{a})=\left(v_{1}, v_{2}, \ldots, v_{\ell} ; a_{0}, a_{1}, \ldots, a_{\ell}\right)$ of shape $\lambda$, define a piecewise linear, continuous map $\pi:[0,1] \rightarrow \mathfrak{h}_{\mathbb{R}}^{*}$ by

$$
\pi(t)=\sum_{p=1}^{q-1}\left(a_{p}-a_{p-1}\right) v_{p}+\left(t-a_{q-1}\right) v_{q} \quad \text { for } a_{q-1} \leq t \leq a_{q}, \quad 1 \leq q \leq \ell .
$$

Clearly, distinct LS paths give rise to distinct piecewise linear functions with values in $\mathfrak{h}_{\mathbb{R}}^{*}$, and we shall make this identification freely in what follows.

Given $\xi \in P^{+}$, we say that an LS path $\pi$ of shape $\lambda$ is $\xi$-dominant if

$$
(\xi+\pi(t))\left(h_{i}\right) \geq 0
$$

for all $i \in I$ and $t \in[0,1]$. Note that $\pi=\left(v_{1}, v_{2}, \ldots, v_{\ell} ; a_{0}, a_{1}, \ldots, a_{\ell}\right)$ is $\xi$ dominant if and only if $\left(\xi+\pi\left(a_{p}\right)\right)\left(h_{i}\right) \geq 0$ for all $i \in I$ and $0 \leq p \leq \ell$.

For $\lambda, \xi, \mu \in P^{+}$, set

$$
\mathbb{B}(\lambda)_{\xi \text {-dom }}^{\mu}=\{\pi \in \mathbb{B}(\lambda): \pi \text { is } \xi \text {-dominant, and } \xi+\pi(1)=\mu\} .
$$


Theorem [Littelmann 1994]. For $\lambda, \xi, \mu \in P^{+}$, we have

$$
\operatorname{dim} \operatorname{Hom}_{\mathfrak{g}}(V(\mu), V(\xi) \otimes V(\lambda))=\# \mathbb{B}(\lambda)_{\xi \text {-dom }}^{\mu} .
$$

4.4. The first step in the proof of the proposition in 4.1 is to describe the set $\mathbb{B}\left(N \omega_{i}\right)$ explicitly when $\omega_{i}$ is minuscule.

Lemma. Let $i \in I$ be such that $\omega_{i}$ is minuscule. Consider a pair $(\underline{v} ; \underline{a})$, where $\underline{v}=\left(v_{1}>v_{2}>\cdots>v_{\ell}\right)$ is a sequence of elements in $W\left(N \omega_{i}\right)$ and

$$
\underline{a}=\left(0=a_{0}<a_{1}<\cdots<a_{\ell}=1\right)
$$

is a sequence of rational numbers (for some $\ell \geq 1$ ). Then we have

$$
(\underline{v} ; \underline{a}) \in \mathbb{B}\left(N \omega_{i}\right) \Longleftrightarrow N a_{p} \in \mathbb{Z}_{+} \text {for all } 0 \leq p \leq \ell .
$$

Proof. Suppose first that $(\underline{v} ; \underline{a})$ is such that $N a_{p} \in \mathbb{Z}_{+}$for all $0 \leq p \leq \ell$, in which case we must prove that for $1 \leq p \leq \ell-1$, there exists an $a_{p}$-chain for $\left(v_{p}, v_{p+1}\right)$. Since $v_{p}>v_{p+1}$, there exists a sequence $v_{p}=\xi_{0}>\xi_{1}>\cdots>\xi_{m}=v_{p+1}$ of elements in $W\left(N \omega_{i}\right)$ such that

$$
\operatorname{dist}\left(\xi_{q-1}, \xi_{q}\right)=1, \quad \xi_{q}=s_{\beta_{q}}\left(\xi_{q-1}\right), \quad \xi_{q-1}\left(h_{\beta_{q}}\right)<0, \quad 1 \leq q \leq m .
$$

Writing $\xi_{p-1}=\boldsymbol{w}\left(N \omega_{i}\right)$ with some $\boldsymbol{w} \in W$, we get

$$
\xi_{p-1}\left(h_{\beta_{p}}\right)=N \underbrace{\left(\boldsymbol{w} \omega_{i}\right)\left(h_{\beta_{p}}\right)}_{\in \mathbb{Z}} \in N \mathbb{Z}_{<0},
$$

which gives $a_{p} \xi_{p-1}\left(h_{\beta_{p}}\right) \in \mathbb{Z}$, as required.

Now suppose that $(\underline{v} ; \underline{a})=\left(v_{1}, v_{2}, \ldots, v_{\ell} ; a_{0}, a_{1}, \ldots, a_{\ell}\right) \in \mathbb{B}\left(N \omega_{i}\right)$. If $v \in$ $W\left(N \omega_{i}\right)$, then since $\omega_{i}$ is minuscule, we have that $v\left(h_{\beta}\right) \in\{0, \pm N\}$ for all $\beta \in R$. We have to prove that $N a_{p} \in \mathbb{Z}_{+}$for $1 \leq p \leq \ell$. The assertion is obvious when $p=0$ or $\ell$. If $1 \leq p \leq \ell-1$, choose an $a_{p}$-chain $v_{p}=\xi_{0}>\xi_{1}>\cdots>\xi_{m}=v_{p+1}$. Then there exists a positive root $\beta$ such that

$$
\xi_{1}=s_{\beta}\left(\xi_{0}\right), \quad a_{p} \xi_{0}\left(h_{\beta}\right) \in \mathbb{Z}_{<0} .
$$

In particular, we have $\xi_{0}\left(h_{\beta}\right)<0$, which implies that $\xi_{0}\left(h_{\beta}\right)=-N$. Thus we get $N a_{p} \in \mathbb{Z}_{+}$as required.

4.5. The following observations are trivial but useful:

$$
\left\{\begin{array}{l}
v \in W\left(r \omega_{i}\right) \Longrightarrow v^{\prime}=\frac{r+1}{r} v \in W\left((r+1) \omega_{i}\right), \\
v, \gamma \in W\left(r \omega_{i}\right), v>\gamma \Rightarrow v^{\prime}>\gamma^{\prime}, \\
0 \leq a<1 \Longrightarrow 0 \leq a^{\prime}=\frac{r a}{r+1}<1, \quad(r+1) a^{\prime} \in \mathbb{Z}_{+} .
\end{array}\right.
$$


Given $(\underline{v} ; \underline{a})=\left(v_{1}, \ldots, v_{\ell} ; a_{0}, \ldots, a_{\ell}\right)$, set

$$
\left(\underline{v}^{\prime} ; \underline{a}^{\prime}\right)= \begin{cases}\left(v_{1}^{\prime}, \ldots, v_{\ell}^{\prime},(r+1) \omega_{i} ; a_{0}^{\prime}, \ldots, a_{\ell}^{\prime}, 1\right) & \text { if } v_{\ell} \neq r \omega_{i}, \\ \left(v_{1}^{\prime}, \ldots, v_{\ell-1}^{\prime},(r+1) \omega_{i} ; a_{0}^{\prime}, \ldots, a_{\ell-1}^{\prime}, 1\right) & \text { if } v_{\ell}=r \omega_{i} .\end{cases}
$$

We now prove the proposition in 4.1 by showing that for each $\mu \in P^{+}$, the assignment

$$
(\underline{\nu} ; \underline{a}) \rightarrow\left(\underline{\nu^{\prime}} ; \underline{a^{\prime}}\right)
$$

gives an injective map

$$
\iota_{r}: \mathbb{B}\left(r \omega_{i}\right)_{s \omega_{i} \text {-dom }}^{\mu} \hookrightarrow \mathbb{B}\left((r+1) \omega_{i}\right)_{(s-1) \omega_{i} \text {-dom }}^{\mu}
$$

It is immediate from the lemma in 4.4, along with (4-4) and the fact that $(r+1) \omega_{i}$ is the minimum element in $W\left((r+1) \omega_{i}\right)$ (with respect to the ordering $>$; see also [Littelmann 1995, Remark 4.2]), that

$$
(\underline{v} ; \underline{a}) \in \mathbb{B}\left(r \omega_{i}\right) \Longrightarrow\left(\underline{\nu^{\prime}} ; \underline{a^{\prime}}\right) \in \mathbb{B}\left((r+1) \omega_{i}\right) .
$$

Let $\pi$ and $\pi^{\prime}$ be the piecewise linear paths associated to $(\underline{v} ; \underline{a})$ and $\left(\underline{v^{\prime}} ; \underline{a^{\prime}}\right)$, respectively (see (4-1)). We have

$$
\pi^{\prime}(t)=\left\{\begin{array}{cc}
\pi\left(\frac{r+1}{r} t\right) & \text { for } 0 \leq t \leq \frac{r}{r+1}, \\
\pi(1)+\left(t-\frac{r}{r+1}\right)(r+1) \omega_{i} & \text { for } \frac{r}{r+1} \leq t \leq 1 .
\end{array}\right.
$$

This proves immediately that

$s \omega_{i}+\pi(1)=\mu \Longrightarrow(s-1) \omega_{i}+\pi^{\prime}(1)=(s-1) \omega_{i}+\pi(1)+\omega_{i}=s \omega_{i}+\pi(1)=\mu$.

Moreover, since

$$
t \in\left[0, \frac{r}{r+1}\right] \Longleftrightarrow \frac{r+1}{r} t \in[0,1],
$$

it follows also that if $\eta$ corresponds to an element of $\mathbb{B}\left(s \omega_{i}\right)$ different from $(\underline{v} ; \underline{a})$, then there exists $t \in[0, r /(r+1)]$ such that

$$
\pi^{\prime}(t) \neq \eta^{\prime}(t)
$$

Thus we have proved that $\iota_{r}$ is injective.

It remains to show that $\pi^{\prime}$ is $(s-1) \omega_{i}$-dominant. Let $j \in I$. If $j \neq i$, we have

$$
\left((s-1) \omega_{i}+\pi^{\prime}(t)\right)\left(h_{j}\right)=\left(\pi^{\prime}(t)\right)\left(h_{j}\right) .
$$

Since $\pi$ is $s \omega_{i}$-dominant, we have

$$
0 \leq\left(s \omega_{i}+\pi(t)\right)\left(h_{j}\right)=(\pi(t))\left(h_{j}\right) \text { for all } 0 \leq t \leq 1 .
$$


Thus, by (4-5), we see that $\left(\pi^{\prime}(t)\right)\left(h_{j}\right) \geq 0$ for all $0 \leq t \leq r /(r+1)$. Also, for $r /(r+1) \leq t \leq 1$, we have

$$
\left(\pi^{\prime}(t)\right)\left(h_{j}\right)=(\pi(1))\left(h_{j}\right)+\left(t-\frac{r}{r+1}\right)(r+1) \underbrace{\omega_{i}\left(h_{j}\right)}_{=0}=(\pi(1))\left(h_{j}\right) \geq 0 .
$$

Thus we have shown that if $j \neq i$, then $\left((s-1) \omega_{i}+\pi^{\prime}(t)\right)\left(h_{j}\right) \geq 0$ for all $0 \leq t \leq 1$.

Next, assume that $j=i$. We see from (4-5) that the function

$$
\left((s-1) \omega_{i}+\pi^{\prime}(t)\right)\left(h_{i}\right)
$$

is strictly increasing on $[r /(r+1), 1]$. Thus it suffices to show that

$$
\left((s-1) \omega_{i}+\pi^{\prime}(t)\right)\left(h_{i}\right) \geq 0 \quad \text { for all } 0 \leq t \leq \frac{r}{r+1} .
$$

Let $0 \leq q \leq \ell$. We have

$$
\left(\pi^{\prime}\left(a_{q}^{\prime}\right)\right)\left(h_{i}\right)=\sum_{p=1}^{q}\left(a_{p}^{\prime}-a_{p-1}^{\prime}\right) v_{p}^{\prime}\left(h_{i}\right) .
$$

Here, we note that $v_{p}^{\prime}\left(h_{i}\right) \in\{0, \pm(r+1)\}$ since $\omega_{i}$ is assumed to be minuscule. Hence,

$$
\begin{aligned}
\left(\pi^{\prime}\left(a_{q}^{\prime}\right)\right)\left(h_{i}\right) & =\sum_{p=1}^{q}(\underbrace{a_{p}^{\prime}-a_{p-1}^{\prime}}_{>0}) v_{p}^{\prime}\left(h_{i}\right) \geq-\sum_{p=1}^{q}\left(a_{p}^{\prime}-a_{p-1}^{\prime}\right)(r+1) \\
& =-(r+1) a_{q}^{\prime}=-r a_{q} \geq-r .
\end{aligned}
$$

Thus, for every $0 \leq q \leq \ell$,

$$
\left((s-1) \omega_{i}+\pi^{\prime}\left(a_{q}^{\prime}\right)\right)\left(h_{i}\right) \geq(s-1)-r=s-(r+1) \geq 0 \text { by assumption, }
$$

which implies (4-6). Thus we have proved the proposition.

\section{The poset $P^{+}(\lambda, 2) / \sim$}

As we remarked earlier, it is clear that if $\sigma \in S_{k}$, then $\lambda$ and $\sigma \lambda$ are in the same equivalence class with respect to $\sim$ for all $\lambda \in P^{+}(\lambda, k)$. However, the following example shows that outside $\mathfrak{s l}_{2}$, the equivalence class of $\sim$ is in general bigger than the $S_{k}$-orbit of an element. Suppose that $\mathfrak{g}$ is of type $\mathfrak{s l}_{3}$ and that $k=3$ and $\lambda=3 \omega_{1}+3 \omega_{2}$. Then it is easily seen that

$$
\lambda=\left(\omega_{2}, \omega_{1}+2 \omega_{2}, 2 \omega_{1}\right) \sim \boldsymbol{\mu}=\left(2 \omega_{1}, \omega_{1}, 2 \omega_{1}+\omega_{2}\right),
$$

but clearly $\lambda$ and $\mu$ are not in the same $S_{3}$-orbit. However, when $k=2$, we prove below (see the lemma in 5.5) that for all simple Lie algebras, the equivalence class is exactly the $S_{2}$-orbit. 
5.1. We begin with an equivalent formulation of the preorder in the case $k=2$.

Proposition. Let $\mathfrak{g}$ be a finite-dimensional simple complex Lie algebra and let $\lambda=\left(\lambda_{1}, \lambda_{2}\right)$ and $\boldsymbol{\mu}=\left(\mu_{1}, \mu_{2}\right)$ be elements of $P^{+}(\lambda, 2) / \sim$ for some $\lambda \in P^{+}$. Then

$$
\begin{aligned}
\lambda \preceq \mu & \Longleftrightarrow\left(\lambda_{1}-\mu_{1}\right)\left(h_{\alpha}\right)\left(\mu_{1}-\lambda_{2}\right)\left(h_{\alpha}\right) \geq 0 \quad \text { for all } \alpha \in R^{+} \\
& \Longleftrightarrow\left(\lambda_{1}-\mu_{1}\right)\left(h_{\alpha}\right)\left(\mu_{1}-\lambda_{2}\right)\left(h_{\alpha}\right) \geq 0 \quad \text { for all } \alpha \in R .
\end{aligned}
$$

In particular, if $\boldsymbol{w} \in W$ is such that $\boldsymbol{w}\left(\lambda_{1}-\lambda_{2}\right) \in P^{+}$, then

$$
\lambda \preceq \boldsymbol{\mu} \Longleftrightarrow \boldsymbol{w}\left(\lambda_{1}-\mu_{1}\right) \in P^{+} \text {and } \boldsymbol{w}\left(\mu_{1}-\lambda_{2}\right) \in P^{+} .
$$

Proof. Since $r_{\alpha, 2}(\boldsymbol{\mu})=\lambda\left(h_{\alpha}\right)$, we see that

$$
r_{\alpha, 2}(\boldsymbol{\mu})-2 r_{\alpha, 1}(\boldsymbol{\mu})= \begin{cases}\left(\mu_{1}-\mu_{2}\right)\left(h_{\alpha}\right) & \text { if } \mu_{2}\left(h_{\alpha}\right) \leq \mu_{1}\left(h_{\alpha}\right), \\ \left(\mu_{2}-\mu_{1}\right)\left(h_{\alpha}\right) & \text { otherwise }\end{cases}
$$

or in other words that

$$
r_{\alpha, 2}(\boldsymbol{\mu})-2 r_{\alpha, 1}(\boldsymbol{\mu})=\left|\left(\mu_{1}-\mu_{2}\right)\left(h_{\alpha}\right)\right| .
$$

Since $k=2$, we see that

$$
\lambda \preceq \boldsymbol{\mu} \Longleftrightarrow r_{\alpha, 1}(\boldsymbol{\lambda}) \leq r_{\alpha, 1}(\boldsymbol{\mu}) \quad \text { for all } \alpha \in R^{+},
$$

and hence we get

$$
\begin{aligned}
\lambda \preceq \boldsymbol{\mu} & \Longleftrightarrow r_{\alpha, 2}(\boldsymbol{\mu})-2 r_{\alpha, 1}(\boldsymbol{\mu}) \leq r_{\alpha, 2}(\boldsymbol{\lambda})-2 r_{\alpha, 1}(\lambda) \\
& \Longleftrightarrow\left|\left(\mu_{1}-\mu_{2}\right)\left(h_{\alpha}\right)\right| \leq\left|\left(\lambda_{1}-\lambda_{2}\right)\left(h_{\alpha}\right)\right| \\
& \Longleftrightarrow\left(\mu_{1}-\mu_{2}\right)\left(h_{\alpha}\right)^{2} \leq\left(\lambda_{1}-\lambda_{2}\right)\left(h_{\alpha}\right)^{2} \\
& \Longleftrightarrow\left(2 \mu_{1}-\lambda_{1}-\lambda_{2}\right)\left(h_{\alpha}\right)^{2} \leq\left(\lambda_{1}-\lambda_{2}\right)\left(h_{\alpha}\right)^{2} \\
& \Longleftrightarrow\left(\lambda_{1}-\mu_{1}\right)\left(h_{\alpha}\right)\left(\mu_{1}-\lambda_{2}\right)\left(h_{\alpha}\right) \geq 0
\end{aligned}
$$

for all $\alpha \in R^{+}$. Since $h_{-\alpha}=-h_{\alpha}$, we see that

$$
\lambda \preceq \boldsymbol{\mu} \Longleftrightarrow\left(\lambda_{1}-\mu_{1}\right)\left(h_{\alpha}\right)\left(\mu_{1}-\lambda_{2}\right)\left(h_{\alpha}\right) \geq 0 \text { for all } \alpha \in R .
$$

Now let $\boldsymbol{w} \in W$ be such that $\boldsymbol{w}\left(\lambda_{1}-\lambda_{2}\right) \in P^{+}$. If $\lambda \preceq \boldsymbol{\mu}$, then for all $\alpha \in R^{+}$,

$$
\boldsymbol{w}\left(\lambda_{1}-\mu_{1}\right)\left(h_{\alpha}\right) \boldsymbol{w}\left(\mu_{1}-\lambda_{2}\right)\left(h_{\alpha}\right)=\left(\lambda_{1}-\mu_{1}\right)\left(h_{\boldsymbol{w}^{-1} \alpha}\right)\left(\mu_{1}-\lambda_{2}\right)\left(h_{\boldsymbol{w}^{-1} \alpha}\right) \geq 0
$$

by the first statement of the proposition. Also, we have

$$
\boldsymbol{w}\left(\lambda_{1}-\mu_{1}\right)\left(h_{\alpha}\right)+\boldsymbol{w}\left(\mu_{1}-\lambda_{2}\right)\left(h_{\alpha}\right)=\boldsymbol{w}\left(\lambda_{1}-\lambda_{2}\right)\left(h_{\alpha}\right) \geq 0 \quad \text { for all } \alpha \in R^{+},
$$

since $\boldsymbol{w}\left(\lambda_{1}-\lambda_{2}\right) \in P^{+}$by assumption. Thus we conclude that $\boldsymbol{w}\left(\lambda_{1}-\mu_{1}\right)\left(h_{\alpha}\right) \geq 0$ and $\boldsymbol{w}\left(\mu_{1}-\lambda_{2}\right)\left(h_{\alpha}\right) \geq 0$ for all $\alpha \in R^{+}$, which implies that both of $\boldsymbol{w}\left(\lambda_{1}-\mu_{1}\right)$ 
and $\boldsymbol{w}\left(\mu_{1}-\lambda_{2}\right)$ are dominant. Conversely, assume that both of $\boldsymbol{w}\left(\lambda_{1}-\mu_{1}\right)$ and $\boldsymbol{w}\left(\mu_{1}-\lambda_{2}\right)$ are dominant. Then, for all $\alpha \in R$,

$$
\left(\lambda_{1}-\mu_{1}\right)\left(h_{\alpha}\right)\left(\mu_{1}-\lambda_{2}\right)\left(h_{\alpha}\right)=\boldsymbol{w}\left(\lambda_{1}-\mu_{1}\right)\left(h_{w^{-1} \alpha}\right) \boldsymbol{w}\left(\mu_{1}-\lambda_{2}\right)\left(h_{w^{-1} \alpha}\right) \geq 0 .
$$

Hence, by the first statement of the proposition, we have $\lambda \preceq \mu$. Thus the second statement of the proposition is established.

5.2. The next result gives information about the maximal elements in $P^{+}(\lambda, 2) / \sim$. Lemma. Let $\lambda \in P^{+}$and let $i \in I, w \in W$ be such that $\lambda-w^{-1} \omega_{i} \in P^{+}$. Then the equivalence classes of $(\lambda, \lambda)$ and $\left(\lambda, \lambda-w^{-1} \omega_{i}\right)$ are maximal in the posets $P^{+}(2 \lambda, 2)$ and $P^{+}\left(2 \lambda-w^{-1} \omega_{i}, 2\right)$, respectively.

Proof. Let $\boldsymbol{\mu}=\left(\mu_{1}, \mu_{2}\right) \in P^{+}(2 \lambda, 2)$ be such that $(\lambda, \lambda) \preceq \boldsymbol{\mu}$ in $P^{+}(2 \lambda, 2) / \sim$. Using the proposition in 5.1, we get $\lambda-\mu_{1} \in P^{+}$and $\mu_{1}-\lambda \in P^{+}$, which forces $\mu_{1}=\mu_{2}=\lambda$ as required.

Similarly, if $\boldsymbol{\mu} \in P^{+}\left(2 \lambda-\boldsymbol{w}^{-1} \omega_{i}, 2\right) / \sim$ with $\left(\lambda, \lambda-\boldsymbol{w}^{-1} \omega_{i}\right) \preceq \boldsymbol{\mu}$, then the proposition in 5.1 gives $\boldsymbol{w}\left(\lambda-\mu_{1}\right) \in P^{+}$and $\boldsymbol{w}\left(\mu_{1}-\lambda\right)+\omega_{i} \in P^{+}$. But this is only possible if either $\mu_{1}=\lambda_{1}$ or $\mu_{1}-\lambda_{1}=-w^{-1} \omega_{i}$. In either case, this implies that $\boldsymbol{\mu}=\left(\lambda, \lambda-\boldsymbol{w}^{-1} \omega_{i}\right)$ in $P^{+}\left(2 \lambda-\boldsymbol{w}^{-1} \omega_{i}, 2\right) / \sim$.

5.3. Suppose that $\mathfrak{g}$ is of type $A_{n}$. Then we can refine the preceding result as follows. Given $\lambda=\sum_{i=1}^{n} r_{i} \omega_{i} \in P^{+}$, define elements $\lambda^{s}, s=1,2$ as follows. If $r_{i} \in 2 \mathbb{Z}_{+}$for all $i \in I$, then take $\lambda^{1}=\lambda^{2}=\lambda$. Otherwise let $1 \leq i_{0}<i_{1}<\cdots<i_{p} \leq n$ be the set where $r_{i}$ is odd and set $I_{+}=I \backslash\left\{i_{0}, \ldots, i_{p}\right\}$. Define

$$
\lambda^{1}=\sum_{s=0}^{p}\left(\frac{r_{i_{s}}+(-1)^{s}}{2}\right) \omega_{i_{s}}+\sum_{i \in I_{+}}\left(\frac{r_{i}}{2}\right) \omega_{i}, \quad \lambda^{2}=\lambda-\lambda^{1} .
$$

In either case, set $\lambda_{\max }=\left(\lambda^{1}, \lambda^{2}\right)$.

Proposition. Let $\lambda \in P^{+}$and $\mathfrak{g}$ be of type $A_{n}$. Then either $\lambda^{1}=\lambda^{2}$ or $\lambda^{2}=$ $\lambda^{1}-\boldsymbol{w}^{-1} \omega_{i}$ for some $\boldsymbol{w} \in W$ and $i \in I$. In either case, $\lambda_{\max }$ is the unique maximal element in $P^{+}(\lambda, 2) / \sim$.

Proof. If $\lambda^{1} \neq \lambda^{2}$, then by definition, we have

$$
\lambda^{1}-\lambda^{2}=\omega_{i_{0}}-\omega_{i_{1}}+\cdots+(-1)^{p} \omega_{i_{p}},
$$

where $0 \leq i_{0}<\cdots<i_{p} \leq n$. It is elementary to see that $\left(\lambda^{1}-\lambda^{2}\right)\left(h_{\alpha}\right) \in\{0, \pm 1\}$, that is, $\lambda^{1}-\lambda^{2}$ is in $W \tau$ for some minuscule $\tau \in P^{+}$, and hence in $W \omega_{i}$ for some $i \in I$. It remains to prove that it is the unique maximal element. In other words, we must prove that if $\boldsymbol{\mu} \in P^{+}(\lambda, 2)$, then $\boldsymbol{\mu} \preceq \lambda_{\max }$. Again, using the proposition in 5.1 , it suffices to prove that

$$
\left(\mu_{1}-\lambda^{1}\right)\left(h_{\alpha}\right)\left(\mu_{1}-\lambda^{2}\right)\left(h_{\alpha}\right)=\left(\mu_{1}-\lambda^{1}\right)\left(h_{\alpha}\right)\left(\lambda^{1}-\mu_{2}\right)\left(h_{\alpha}\right) \geq 0 .
$$


If $\left(\mu_{1}-\lambda^{1}\right)\left(h_{\alpha}\right)=0$, there is nothing to prove. If $\left(\mu_{1}-\lambda^{1}\right)\left(h_{\alpha}\right)>0$, then since $\left(\lambda^{1}-\lambda^{2}\right)\left(h_{\alpha}\right) \in\{0, \pm 1\}$, we get $\mu_{1}\left(h_{\alpha}\right) \geq \lambda^{2}\left(h_{\alpha}\right)$ as required. The case when $\left(\mu_{1}-\lambda^{1}\right)\left(h_{\alpha}\right)<0$ is identical.

\section{4 .}

Proposition. Let $\lambda, \boldsymbol{\mu} \in P^{+}(\lambda, 2) / \sim$ with $\lambda \prec \boldsymbol{\mu}$ and assume there exist $\boldsymbol{w} \in W$ and $i_{0} \in I$ such that $\boldsymbol{w}\left(\lambda_{1}-\lambda_{2}\right) \in P^{+}$and

$$
\boldsymbol{w}\left(\lambda_{1}-\mu_{1}\right)\left(h_{i_{0}}\right) \boldsymbol{w}\left(\mu_{1}-\lambda_{2}\right)\left(h_{i_{0}}\right)>0 .
$$

Then $\left(\lambda_{1}-\boldsymbol{w}^{-1} \omega_{i_{0}}, \lambda_{2}+\boldsymbol{w}^{-1} \omega_{i_{0}}\right) \in P^{+}(\lambda, 2)$ and

$$
\lambda \prec\left(\lambda_{1}-\boldsymbol{w}^{-1} \omega_{i_{0}}, \lambda_{2}+\boldsymbol{w}^{-1} \omega_{i_{0}}\right) \preceq \boldsymbol{\mu} .
$$

Proof. First we remark that by (5-2) and the assumption that $\boldsymbol{w}\left(\lambda_{1}-\lambda_{2}\right) \in P^{+}$,

$$
\boldsymbol{w}\left(\lambda_{1}-\lambda_{2}\right)\left(h_{i_{0}}\right)=\boldsymbol{w}\left(\lambda_{1}-\mu_{1}\right)\left(h_{i_{0}}\right)+\boldsymbol{w}\left(\mu_{1}-\lambda_{2}\right)\left(h_{i_{0}}\right)>0 .
$$

Let us show that $\lambda_{1}-\boldsymbol{w}^{-1} \omega_{i_{0}}$ and $\lambda_{2}+\boldsymbol{w}^{-1} \omega_{i_{0}}$ are dominant, which implies that $\left(\lambda_{1}-\boldsymbol{w}^{-1} \omega_{i_{0}}, \lambda_{2}+\boldsymbol{w}^{-1} \omega_{i_{0}}\right) \in P^{+}(\lambda, 2)$. For $j \in I$, write

$$
\boldsymbol{w} h_{j}=\sum_{i=1}^{n} r_{i} h_{i},
$$

and note that either $r_{i} \geq 0$ for all $i \in I$ or $r_{i} \leq 0$ for all $i \in I$. If $r_{i_{0}} \leq 0$, then obviously $\left(\lambda_{1}-w^{-1} \omega_{i_{0}}\right)\left(h_{j}\right) \geq 0$. If $r_{i} \geq 0$ for all $i \in I$, then we have

$$
\left(\lambda_{1}-\lambda_{2}\right)\left(h_{j}\right)=\boldsymbol{w}\left(\lambda_{1}-\lambda_{2}\right)\left(w h_{j}\right) \geq r_{i_{0}} \boldsymbol{w}\left(\lambda_{1}-\lambda_{2}\right)\left(h_{i_{0}}\right) \geq r_{i_{0}},
$$

where the first inequality follows from the assumption that $w\left(\lambda_{1}-\lambda_{2}\right) \in P^{+}$, and the second inequality follows from (5-4). Hence $\lambda_{1}\left(h_{j}\right) \geq \lambda_{2}\left(h_{j}\right)+r_{i_{0}} \geq r_{i_{0}}$, and hence $\left(\lambda_{1}-\boldsymbol{w}^{-1} \omega_{i_{0}}\right)\left(h_{j}\right) \geq 0$, since $\boldsymbol{w}^{-1} \omega_{i_{0}}\left(h_{j}\right)=r_{i_{0}}$. Thus we have proved that $\lambda_{1}-\boldsymbol{w}^{-1} \omega_{i_{0}} \in P^{+}$. To prove that $\lambda_{2}+\boldsymbol{w}^{-1} \omega_{i_{0}} \in P^{+}$, we note that if $r_{i_{0}} \geq 0$, there is nothing to prove. If $r_{i} \leq 0$ for all $i \in I$, then we have

$$
\left(\lambda_{1}-\lambda_{2}\right)\left(h_{j}\right)=\boldsymbol{w}\left(\lambda_{1}-\lambda_{2}\right)\left(w h_{j}\right) \leq r_{i_{0}} \boldsymbol{w}\left(\lambda_{1}-\lambda_{2}\right)\left(h_{i_{0}}\right) \leq r_{i_{0}},
$$

where the first inequality follows from the assumption that $\boldsymbol{w}\left(\lambda_{1}-\lambda_{2}\right) \in P^{+}$, and the second inequality follows from (5-4). Hence $\lambda_{2}\left(h_{j}\right) \geq \lambda_{1}\left(h_{j}\right)-r_{i_{0}} \geq-r_{i_{0}}$, and so $\left(\lambda_{2}+\boldsymbol{w}^{-1} \omega_{i_{0}}\right)\left(h_{j}\right)=\lambda_{2}\left(h_{j}\right)+r_{i_{0}} \geq 0$, proving that $\lambda_{2}+\boldsymbol{w}^{-1} \omega_{i} \in P^{+}$.

By the proposition in 5.1, we see that $\boldsymbol{w}\left(\lambda_{1}-\mu_{1}\right)$ and $\boldsymbol{w}\left(\mu_{1}-\lambda_{2}\right)$ are in $P^{+}$. Hence (5-2) gives

$\boldsymbol{w}\left(\lambda_{1}-\mu_{1}\right)\left(h_{i_{0}}\right)>0, \quad \boldsymbol{w}\left(\mu_{1}-\lambda_{2}\right)\left(h_{i_{0}}\right)>0, \quad$ and hence $\boldsymbol{w}\left(\lambda_{1}-\lambda_{2}\right)\left(h_{i_{0}}\right)>0$, which in turn gives

$$
\boldsymbol{w}\left(\lambda_{1}-\mu_{1}\right)-\omega_{i_{0}}, \quad \boldsymbol{w}\left(\mu_{1}-\lambda_{2}\right)-\omega_{i_{0}}, \quad \boldsymbol{w}\left(\lambda_{1}-\lambda_{2}\right)-\omega_{i_{0}} \in P^{+} .
$$


To prove

$$
\lambda \preceq\left(\lambda_{1}-\boldsymbol{w}^{-1} \omega_{i_{0}}, \lambda_{2}+\boldsymbol{w}^{-1} \omega_{i_{0}}\right) \preceq \boldsymbol{\mu},
$$

we must show that for all $\alpha \in R^{+}$,

$$
\begin{aligned}
\boldsymbol{w}^{-1} \omega_{i_{0}}\left(h_{\alpha}\right)\left(\lambda_{1}-\boldsymbol{w}^{-1} \omega_{i_{0}}-\lambda_{2}\right)\left(h_{\alpha}\right) & \geq 0, \\
\left(\lambda_{1}-\boldsymbol{w}^{-1} \omega_{i_{0}}-\mu_{1}\right)\left(h_{\alpha}\right)\left(\mu_{1}-\lambda_{2}-\boldsymbol{w}^{-1} \omega_{i_{0}}\right)\left(h_{\alpha}\right) & \geq 0,
\end{aligned}
$$

or equivalently that

$$
\begin{array}{r}
\omega_{i_{0}}\left(h_{\alpha}\right)\left(\boldsymbol{w}\left(\lambda_{1}-\lambda_{2}\right)-\omega_{i_{0}}\right)\left(h_{\alpha}\right) \geq 0, \\
\left(\boldsymbol{w}\left(\lambda_{1}-\mu_{1}\right)-\omega_{i_{0}}\right)\left(h_{\alpha}\right)\left(\boldsymbol{w}\left(\mu_{1}-\lambda_{2}\right)-\omega_{i_{0}}\right)\left(h_{\alpha}\right) \geq 0 .
\end{array}
$$

But this is now immediate from (5-5).

In order to prove (5-3), it remains to show $\lambda \prec\left(\lambda_{1}-\boldsymbol{w}^{-1} \omega_{i_{0}}, \lambda_{2}+\boldsymbol{w}^{-1} \omega_{i_{0}}\right)$. For that, notice that $\lambda_{1}-\boldsymbol{w}^{-1} \omega_{i_{0}} \notin\left\{\lambda_{1}, \lambda_{2}\right\}$, since then by the lemma in 5.2, we would have that $\lambda$ is a maximal element of $P^{+}(\lambda, 2)$, and this would contradict the fact that $\lambda \prec \mu$.

5.5. In this section, we will show that for $k=2$, equivalence classes in $P^{+}(\lambda, 2)$ are the $S_{2}$-orbits, generalizing the results of Section 3.2.

Lemma. Let $\mathfrak{g}$ be arbitrary and let $\lambda, \boldsymbol{\mu} \in P^{+}(\lambda, 2)$ for some $\lambda \in P^{+}$. Then $\boldsymbol{\lambda} \sim \boldsymbol{\mu}$ if and only if $\boldsymbol{\mu}$ and $\lambda$ are in the same $S_{2}$-orbit.

Proof. Suppose that $\lambda=\left(\lambda_{1}, \lambda_{2}\right)$ and $\mu=\left(\mu_{1}, \mu_{2}\right)$ and set $v=\lambda_{1}-\lambda_{2}$ and $v^{\prime}=\mu_{1}-\mu_{2}$. If $\lambda \sim \boldsymbol{\mu}$ then we see from (5-1) that for all $\alpha \in R$, we have $v\left(h_{\alpha}\right)= \pm v^{\prime}\left(h_{\alpha}\right)$, where the sign depends on $\alpha$. It suffices to show that we can choose the sign consistently. Suppose for a contradiction that this is not so; then there exist a connected subset $I_{0}$ of $I$ and $i_{1}, i_{2} \in I_{0}$ such that

$$
\begin{aligned}
& v\left(h_{i_{1}}\right)=v^{\prime}\left(h_{i_{1}}\right) \neq 0, \\
& v\left(h_{i_{2}}\right)=-v^{\prime}\left(h_{i_{2}}\right) \neq 0, \\
& v\left(h_{j}\right)=v^{\prime}\left(h_{j}\right)=0, \quad j \in I_{0} \backslash\left\{i_{1}, i_{2}\right\} .
\end{aligned}
$$

Set $\beta=\sum_{i \in I_{0}} \alpha_{i}$; we can easily check that $\beta$ is a (positive) root, that is, $\beta \in R^{+}$. Then

$$
v\left(h_{\beta}\right)=v\left(h_{i_{1}}\right)+v\left(h_{i_{2}}\right)=v^{\prime}\left(h_{i_{1}}\right)-v^{\prime}\left(h_{i_{2}}\right) \neq \pm\left(v^{\prime}\left(h_{i_{1}}\right)+v^{\prime}\left(h_{i_{2}}\right)\right) .
$$

Since $\beta \in R^{+}$, we get the required contradiction.

\section{Proof of Theorem 1(iii)}

In this section, we assume that $\mathfrak{g}$ is of type $A_{2}$ and prove Theorem 1(iii). We begin by showing that we can restrict our attention to certain elements $\lambda$ and $\mu$ of $P^{+}(\lambda, 2)$. 
6.1. Since the poset $P^{+}(\lambda, 2)$ is finite, it suffices to prove part (iii) of Theorem 1 for $\lambda$ and $\boldsymbol{\mu}$, where $\boldsymbol{\mu}$ is a cover of $\lambda$, that is, $\lambda \prec \boldsymbol{\mu}$ and there does not exist $\boldsymbol{v} \in P^{+}(\lambda, 2)$ with $\lambda \prec \boldsymbol{v} \prec \boldsymbol{\mu}$. We first show that in fact it suffices to prove Theorem 1(iii) for certain special $\lambda$ and also that for these $\lambda$ we can restrict our attention to certain special covers.

We shall use freely the following two facts. The first is well known.

$$
V(\lambda) \otimes V(\mu) \cong_{\mathfrak{g}} V(\mu) \otimes V(\lambda) .
$$

The second fact is that the partial order on $P^{+}(\lambda, k)$ is compatible with duals (see (1-1) and (2-2)) and that for all $\lambda, \mu, v \in P^{+}$, we have

$\operatorname{dim} \operatorname{Hom}_{\mathfrak{g}}(V(v), V(\lambda) \otimes V(\mu))=\operatorname{dim} \operatorname{Hom}_{\mathfrak{g}}\left(V\left(-\boldsymbol{w}_{0} v\right), V\left(-\boldsymbol{w}_{0} \lambda\right) \otimes V\left(-\boldsymbol{w}_{0} \mu\right)\right)$.

This allows us to switch freely between proving Theorem 1(iii) either for $\boldsymbol{\lambda} \prec \boldsymbol{\mu}$ or for $-\boldsymbol{w}_{0} \boldsymbol{\lambda} \prec-\boldsymbol{w}_{0} \boldsymbol{\mu}$. Recall that $-\boldsymbol{w}_{0} \omega_{1}=\omega_{2}$.

Proposition. Let $\lambda \in P^{+}$and $\lambda=\left(\lambda_{1}, \lambda_{2}\right) \in P^{+}(\lambda, 2)$ and assume that $\boldsymbol{\mu}=\left(\mu_{1}, \mu_{2}\right)$ covers $\lambda$. It suffices to prove Theorem 1(iii) is true when $\lambda$ and $\boldsymbol{\mu}$ satisfy the following conditions for some $\boldsymbol{w} \in\left\{\mathrm{id}, \boldsymbol{s}_{1}, \boldsymbol{s}_{2}\right\}$ :

$$
\boldsymbol{w}\left(\lambda_{1}-\lambda_{2}\right) \in P^{+}, \quad \boldsymbol{w}\left(\lambda_{1}-\lambda_{2}\right)\left(h_{1}\right)>0,
$$

and either

$$
\left\{\begin{array}{l}
\boldsymbol{\mu}=\left(\lambda_{1}-\boldsymbol{w} \omega_{1}, \lambda_{2}+\boldsymbol{w} \omega_{1}\right) \quad \text { or } \\
\boldsymbol{\mu}=\left(\lambda_{1}-\boldsymbol{w}\left(\lambda_{1}-\lambda_{2}\right)\left(h_{1}\right) \boldsymbol{w} \omega_{1}, \lambda_{2}+\boldsymbol{w}\left(\lambda_{1}-\lambda_{2}\right)\left(h_{1}\right) \boldsymbol{w} \omega_{1}\right) .
\end{array}\right.
$$

Proof. We first prove that we can assume that $\lambda$ satisfies the conditions in (6-1). Suppose that $\lambda=\left(\lambda_{1}, \lambda_{2}\right) \in P^{+}(\lambda, 2)$ is such that $\lambda_{1}-\lambda_{2} \in P^{+}$but $\lambda_{1}\left(h_{1}\right)=\lambda_{2}\left(h_{1}\right)$. Since $\lambda$ is not the maximal element in $P^{+}(\lambda, 2)$, it follows from the lemma in 5.2 that $\lambda_{1} \neq \lambda_{2}$, and hence we must have $\lambda_{1}\left(h_{2}\right)>\lambda_{2}\left(h_{2}\right)$. We have $-\boldsymbol{w}_{0} \boldsymbol{\lambda} \prec-\boldsymbol{w}_{0} \boldsymbol{\mu}$, and hence $-w_{0}\left(\lambda_{1}-\lambda_{2}\right)\left(h_{1}\right)>0$. If $s_{2}\left(\lambda_{1}-\lambda_{2}\right) \in P^{+}$or $s_{1}\left(\lambda_{1}-\lambda_{2}\right) \in P^{+}$, a similar argument shows that either $\lambda$ or $-w_{0} \lambda$ satisfies the conditions in (6-1). Suppose now that $\boldsymbol{w}\left(\lambda_{1}-\lambda_{2}\right) \in P^{+}$but $\boldsymbol{w} \notin\left\{\mathrm{id}, \boldsymbol{s}_{1}, \boldsymbol{s}_{2}\right\}$. Then $\boldsymbol{w} \boldsymbol{w}_{0} \in\left\{\mathrm{id}, \boldsymbol{s}_{1}, \boldsymbol{s}_{2}\right\}$, and hence we can work with the pair $\left(-\boldsymbol{w}_{0} \lambda_{2},-\boldsymbol{w}_{0} \lambda_{1}\right)$.

We now prove that we can also assume that $\boldsymbol{\mu}$ satisfies the conditions in (6-2).

Case 1. Suppose that there exists $i \in I=\{1,2\}$ such that

$$
\boldsymbol{w}\left(\lambda_{1}-\mu_{1}\right)\left(h_{i}\right) \boldsymbol{w}\left(\mu_{1}-\lambda_{2}\right)\left(h_{i}\right)>0,
$$

where $\boldsymbol{w} \in\left\{\mathrm{id}, \boldsymbol{s}_{1}, \boldsymbol{s}_{2}\right\}$. We see from the proposition in 5.1 that $\boldsymbol{w}\left(\lambda_{1}-\mu_{1}\right)$ and $\boldsymbol{w}\left(\mu_{1}-\lambda_{2}\right) \in P^{+}$. Thus we have $\boldsymbol{w}\left(\lambda_{1}-\mu_{1}\right)\left(h_{i}\right)>0$ and $\boldsymbol{w}\left(\mu_{1}-\lambda_{2}\right)\left(h_{i}\right)>0$. In particular,

$$
\boldsymbol{w}\left(\lambda_{1}-\lambda_{2}\right)\left(h_{i}\right)=\boldsymbol{w}\left(\lambda_{1}-\mu_{1}\right)\left(h_{i}\right)+\boldsymbol{w}\left(\mu_{1}-\lambda_{2}\right)\left(h_{i}\right)>0 .
$$


Subcase 1.1. If $i=1$, then it follows from the proposition in 5.4 that

$$
\lambda \prec\left(\lambda_{1}-\boldsymbol{w}^{-1} \omega_{1}, \lambda_{2}+\boldsymbol{w}^{-1} \omega_{1}\right)=\left(\lambda_{1}-\boldsymbol{w} \omega_{1}, \lambda_{2}+\boldsymbol{w} \omega_{1}\right) \preceq \boldsymbol{\mu} .
$$

Since $\boldsymbol{\mu}$ covers $\lambda$, it follows that $\boldsymbol{\mu}=\left(\lambda_{1}-\boldsymbol{w} \omega_{1}, \lambda_{2}+\boldsymbol{w} \omega_{1}\right)$, as required.

Subcase 1.2. If $i=2$, then it follows from the proposition in 5.4 that

$$
\lambda \prec\left(\lambda_{1}-w^{-1} \omega_{2}, \lambda_{2}+w^{-1} \omega_{2}\right)=\left(\lambda_{1}-w \omega_{2}, \lambda_{2}+w \omega_{2}\right) \preceq \mu .
$$

By the "duality", we get

$$
-\boldsymbol{w}_{0} \lambda \prec\left(-\boldsymbol{w}_{0} \lambda_{1}-\left(-\boldsymbol{w}_{0} \boldsymbol{w} \omega_{2}\right),-\boldsymbol{w}_{0} \lambda_{2}+\left(-\boldsymbol{w}_{0} \boldsymbol{w} \omega_{2}\right)\right) \preceq-\boldsymbol{w}_{0} \boldsymbol{\mu} .
$$

Since $-\boldsymbol{w}_{0} \boldsymbol{\mu}$ covers $-\boldsymbol{w}_{0} \boldsymbol{\lambda}$, we get

$$
-\boldsymbol{w}_{0} \boldsymbol{\mu}=\left(-\boldsymbol{w}_{0} \lambda_{1}-\left(-\boldsymbol{w}_{0} \boldsymbol{w} \omega_{2}\right),-\boldsymbol{w}_{0} \lambda_{2}+\left(-\boldsymbol{w}_{0} \boldsymbol{w} \omega_{2}\right)\right) .
$$

We set $\widetilde{\boldsymbol{w}}=\boldsymbol{w}_{0} \boldsymbol{w} \boldsymbol{w}_{0}$ and note that we have

$$
\widetilde{\boldsymbol{w}}:= \begin{cases}\text { id } & \text { if } \boldsymbol{w}=\mathrm{id}, \\ \boldsymbol{s}_{2} & \text { if } \boldsymbol{w}=\boldsymbol{s}_{1}, \\ \boldsymbol{s}_{1} & \text { if } \boldsymbol{w}=\boldsymbol{s}_{2} .\end{cases}
$$

We also have

$$
\begin{aligned}
\widetilde{\boldsymbol{w}}\left(\left(-\boldsymbol{w}_{0} \lambda_{1}\right)-\left(-\boldsymbol{w}_{0} \lambda_{2}\right)\right) & =-\boldsymbol{w}_{0} \boldsymbol{w}\left(\lambda_{1}-\lambda_{2}\right) \in P^{+}, \\
\widetilde{\boldsymbol{w}}\left(\left(-\boldsymbol{w}_{0} \lambda_{1}\right)-\left(-\boldsymbol{w}_{0} \lambda_{2}\right)\right)\left(h_{1}\right) & =-\boldsymbol{w}_{0} \boldsymbol{w}\left(\lambda_{1}-\lambda_{2}\right)\left(h_{1}\right) \\
& =\boldsymbol{w}\left(\lambda_{1}-\lambda_{2}\right)\left(h_{2}\right)>0 \quad \text { by }(6-3) .
\end{aligned}
$$

Hence, $-\boldsymbol{w}_{0} \boldsymbol{\lambda}$ and $-\boldsymbol{w}_{0} \boldsymbol{\mu}$ satisfy the conditions (with $\boldsymbol{w}$ replaced by $\widetilde{\boldsymbol{w}}$ ). Hence, if Theorem 1(iii) is established for this pair, then it follows for the pair $\lambda$ and $\boldsymbol{\mu}$ as discussed earlier.

Case 2. Suppose that

$$
\boldsymbol{w}\left(\lambda_{1}-\mu_{1}\right)\left(h_{i}\right) \boldsymbol{w}\left(\mu_{1}-\lambda_{2}\right)\left(h_{i}\right) \leq 0 \quad \text { for all } i \in I=\{1,2\},
$$

where $w \in\left\{\mathrm{id}, \boldsymbol{s}_{1}, \boldsymbol{s}_{2}\right\}$. We see from the proposition in 5.1 that $w\left(\lambda_{1}-\mu_{1}\right) \in P^{+}$ and $\boldsymbol{w}\left(\mu_{1}-\lambda_{2}\right) \in P^{+}$. Thus,

$$
\boldsymbol{w}\left(\lambda_{1}-\mu_{1}\right)\left(h_{i}\right) \boldsymbol{w}\left(\mu_{1}-\lambda_{2}\right)\left(h_{i}\right)=0 \quad \text { for all } i \in I=\{1,2\},
$$

which implies that $\boldsymbol{w} \mu_{1}\left(h_{i}\right)=\boldsymbol{w} \lambda_{1}\left(h_{i}\right)$ or $\boldsymbol{w} \mu_{1}\left(h_{i}\right)=\boldsymbol{w} \lambda_{2}\left(h_{i}\right)$ for each $i=1,2$. Remark that $\lambda$ is not the maximal element, since $\lambda \prec \mu$. Therefore it follows that the only possibilities are

$$
\boldsymbol{w} \mu_{1}=\left(\boldsymbol{w} \lambda_{2}\right)\left(h_{1}\right) \omega_{1}+\left(\boldsymbol{w} \lambda_{1}\right)\left(h_{2}\right) \omega_{2} \quad \text { or } \quad \boldsymbol{w} \mu_{1}=\left(\boldsymbol{w} \lambda_{1}\right)\left(h_{1}\right) \omega_{1}+\left(\boldsymbol{w} \lambda_{2}\right)\left(h_{2}\right) \omega_{2} .
$$


In turn this implies that

$$
\begin{aligned}
\boldsymbol{\mu} \sim\left(\left(\boldsymbol{w} \lambda_{2}\right)\left(h_{1}\right) \boldsymbol{w}^{-1}\right. & \left.\omega_{1}+\left(\boldsymbol{w} \lambda_{1}\right)\left(h_{2}\right) \boldsymbol{w}^{-1} \omega_{2},\left(\boldsymbol{w} \lambda_{1}\right)\left(h_{1}\right) \boldsymbol{w}^{-1} \omega_{1}+\left(\boldsymbol{w} \lambda_{2}\right)\left(h_{2}\right) \boldsymbol{w}^{-1} \omega_{2}\right) \\
& =\left(\lambda_{1}-\boldsymbol{w}\left(\lambda_{1}-\lambda_{2}\right)\left(h_{1}\right) \boldsymbol{w}^{-1} \omega_{1}, \lambda_{2}+\boldsymbol{w}\left(\lambda_{1}-\lambda_{2}\right)\left(h_{1}\right) \boldsymbol{w}^{-1} \omega_{1}\right) \\
& =\left(\lambda_{1}-\boldsymbol{w}\left(\lambda_{1}-\lambda_{2}\right)\left(h_{1}\right) \boldsymbol{w} \omega_{1}, \lambda_{2}+\boldsymbol{w}\left(\lambda_{1}-\lambda_{2}\right)\left(h_{1}\right) \boldsymbol{w} \omega_{1}\right),
\end{aligned}
$$

as required; here we use the fact that

$$
\left\{\begin{array}{l}
\lambda_{1}=\left(\boldsymbol{w} \lambda_{1}\right)\left(h_{1}\right) \boldsymbol{w}^{-1} \omega_{1}+\left(\boldsymbol{w} \lambda_{1}\right)\left(h_{2}\right) \boldsymbol{w}^{-1} \omega_{2} \\
\lambda_{2}=\left(\boldsymbol{w} \lambda_{2}\right)\left(h_{1}\right) \boldsymbol{w}^{-1} \omega_{1}+\left(\boldsymbol{w} \lambda_{2}\right)\left(h_{2}\right) \boldsymbol{w}^{-1} \omega_{2}
\end{array}\right.
$$

and then the fact that $\boldsymbol{w}^{-1}=\boldsymbol{w}$.

6.2. We now recall from [Kashiwara and Nakashima 1994; Nakashima 1993] a tableaux description of tensor product multiplicities. Given $\lambda \in P^{+}$, let $\mathbb{\mathbb { V }}(\lambda) \subset \mathbb{Z}_{+}^{5}$ be the subset consisting of tuples $\left(s_{1,1}, s_{1,2}, s_{1,3}, s_{2,2}, s_{2,3}\right)$ satisfying the conditions

$$
\begin{array}{rlrl}
s_{1,1}+s_{1,2}+s_{1,3} & =\lambda\left(h_{1}\right)+\lambda\left(h_{2}\right), & & s_{2,2}+s_{2,3}=\lambda\left(h_{2}\right), \\
s_{1,1} \geq s_{2,2}, & s_{1,1}+s_{1,2} \geq s_{2,2}+s_{2,3} .
\end{array}
$$

Then, it is proved in [Kashiwara and Nakashima 1994] that

$$
\operatorname{dim} V(\lambda)=\# \mathbb{T}(\lambda) .
$$

(This is just the number of semistandard tableaux with entries from $\{1,2,3\}$ of shape $\lambda$, where $s_{i, j}$ corresponds to the number of $j$ in the $i$-th row). Moreover, if $v \in P$ and we set

$\mathbb{T}(\lambda)^{v}=\left\{\left(s_{i, j}\right) \in \mathbb{T}(\lambda): s_{1,1}-s_{1,2}-s_{2,2}=v\left(h_{1}\right), s_{1,2}+s_{2,2}-s_{1,3}-s_{2,3}=v\left(h_{2}\right)\right\}$, then

$$
\operatorname{dim} V(\lambda)_{\nu}=\# \mathbb{T}(\lambda)^{v} .
$$

In particular, if $\left(s_{i, j}\right),\left(t_{i, j}\right) \in \mathbb{T}(\lambda)^{v}$, then they satisfy

$$
s_{1,1}=t_{1,1}, \quad s_{1,2}+s_{2,2}=t_{1,2}+t_{2,2}, \quad s_{1,3}+s_{2,3}=t_{1,3}+t_{2,3} .
$$

Suppose now that $\mu, v \in P^{+}$; then [Nakashima 1993]

$$
\operatorname{dim} \operatorname{Hom}_{\mathfrak{g}}(V(v), V(\mu) \otimes V(\lambda))=\# \mathbb{T}(\lambda)_{\mu}^{\nu},
$$

where $\mathbb{T}(\lambda)_{\mu}^{\nu}$ is the subset of $\mathbb{T}(\lambda)^{\nu}$ consisting of $\left(s_{1,1}, s_{1,2}, s_{1,3}, s_{2,2}, s_{2,3}\right) \in \mathbb{T}(\lambda)$ satisfying the following additional constraints:

$$
\begin{aligned}
s_{1,2} & \leq \mu\left(h_{1}\right), \quad s_{1,3} \leq \mu\left(h_{2}\right), \quad s_{2,3}+s_{1,3} \leq \mu\left(h_{2}\right)+s_{1,2}, \\
v\left(h_{1}\right)+v\left(h_{2}\right) & =\mu\left(h_{1}\right)+\mu\left(h_{2}\right)+s_{1,1}-s_{1,3}-s_{2,3}, \\
v\left(h_{2}\right) & =\mu\left(h_{2}\right)+s_{1,2}+s_{2,2}-s_{1,3}-s_{2,3} .
\end{aligned}
$$


As a consequence, we see that to prove Theorem 1(iii), we must prove that if $\lambda, \mu \in P^{+}(\lambda, 2)$, then

$$
\lambda \preceq \mu \Longrightarrow \# \mathbb{T}\left(\lambda_{2}\right)_{\lambda_{1}}^{v} \leq \# \mathbb{T}\left(\mu_{2}\right)_{\mu_{1}}^{v} \quad \text { for each } v \in P^{+} .
$$

This is done in the rest of the section.

6.3. Keep the notation in the proposition in 6.1. In this subsection, we prove that Theorem 1(iii) is true if $\boldsymbol{\lambda}$ and $\boldsymbol{\mu}$ satisfy the conditions (6-1) and (6-2) with $\boldsymbol{w}=\mathrm{id}$ or $\boldsymbol{w}=s_{2}$. By (6-11), it suffices to find an injective map from

$$
\mathbb{T}\left(\lambda_{2}\right)_{\lambda_{1}}^{\nu} \hookrightarrow \mathbb{T}\left(\lambda_{2}+a w \omega_{1}\right)_{\lambda_{1}-a w \omega_{1}}^{\nu}=\mathbb{T}\left(\lambda_{2}+a \omega_{1}\right)_{\lambda_{1}-a \omega_{1}}^{\nu}
$$

for each $v \in P^{+}$, where $a$ equals either 1 or $\boldsymbol{w}\left(\lambda_{1}-\lambda_{2}\right)\left(h_{1}\right)$; note that

$$
\boldsymbol{w}\left(\lambda_{1}-\lambda_{2}\right)\left(h_{1}\right)>0
$$

by the second equality of (6-1). This is obtained as a corollary of the following proposition.

Proposition. Keep the notation above. For each $v \in P^{+}$, there exists $0 \leq \ell \leq a$ such that for all $\left(s_{i, j}\right) \in \mathbb{T}\left(\lambda_{2}\right)_{\lambda_{1}}^{v}$, we have

$$
a-\ell \leq s_{1,2} \leq \lambda_{1}\left(h_{1}\right)-\ell, \quad s_{1,3} \leq \lambda_{1}\left(h_{2}\right)-(a-\ell), \quad s_{2,3} \geq a-\ell .
$$

Proof. First, let us show that

$$
\lambda_{1}\left(h_{1}\right)+\lambda_{1}\left(h_{2}\right)-a \geq \lambda_{2}\left(h_{1}\right)+\lambda_{2}\left(h_{2}\right), \quad \lambda_{1}\left(h_{1}\right)-\lambda_{2}\left(h_{1}\right) \geq a .
$$

Indeed, since $\boldsymbol{w}\left(\lambda_{1}-\lambda_{2}\right)\left(h_{1}\right) \geq a$ by the definition of $a$, we have

$$
\begin{cases}\left(\lambda_{1}-\lambda_{2}\right)\left(h_{1}\right) \geq a & \text { if } \boldsymbol{w}=\mathrm{id}, \\ \left(\lambda_{1}-\lambda_{2}\right)\left(h_{1}+h_{2}\right) \geq a & \text { if } \boldsymbol{w}=\boldsymbol{s}_{2},\end{cases}
$$

which implies the second (resp. first) inequality of (6-13) if $\boldsymbol{w}=\mathrm{id}$ (resp. $\boldsymbol{w}=\boldsymbol{s}_{2}$ ). Also, since $w\left(\lambda_{1}-\lambda_{2}\right) \in P^{+}$, we see that

$$
\boldsymbol{w}\left(\lambda_{1}-\lambda_{2}\right)\left(h_{1}+h_{2}\right) \geq \boldsymbol{w}\left(\lambda_{1}-\lambda_{2}\right)\left(h_{1}\right) \geq a .
$$

Thus we get

$$
\begin{cases}\left(\lambda_{1}-\lambda_{2}\right)\left(h_{1}+h_{2}\right) \geq a & \text { if } \boldsymbol{w}=\mathrm{id}, \\ \left(\lambda_{1}-\lambda_{2}\right)\left(h_{1}\right) \geq a & \text { if } \boldsymbol{w}=\boldsymbol{s}_{2},\end{cases}
$$

which implies the first (resp. second) inequality of (6-13) if $\boldsymbol{w}=\mathrm{id}$ (resp. $\boldsymbol{w}=\boldsymbol{s}_{2}$ ).

By (6-8), we have $t_{1,2} \leq \lambda_{1}\left(h_{1}\right)$ for all $\left(t_{i, j}\right) \in \mathbb{T}\left(\lambda_{2}\right)_{\lambda_{1}}^{v}$. Thus we can choose $0 \leq \ell \leq a$ maximal such that for all $\left(t_{i, j}\right) \in \mathbb{T}\left(\lambda_{2}\right)_{\lambda_{1}}^{\nu}$,

$$
t_{1,2} \leq \lambda_{1}\left(h_{1}\right)-\ell \text {. }
$$

In particular, we can and do fix an element $\left(s_{i, j}\right) \in \mathbb{T}\left(\lambda_{2}\right)_{\lambda_{1}}^{v}$ with $s_{1,2}=\lambda_{1}\left(h_{1}\right)-\ell$. 
Suppose that there exists $\left(t_{i, j}\right) \in \mathbb{T}\left(\lambda_{2}\right)_{\lambda_{1}}^{v}$, with $t_{1,3}>\lambda_{1}\left(h_{2}\right)+\ell-a$. Then (6-6) gives

$$
t_{2,2}+t_{1,2}=s_{2,2}+s_{1,2} \geq s_{1,2}=\lambda_{1}\left(h_{1}\right)-\ell .
$$

This implies that

$$
\begin{aligned}
\lambda_{2}\left(h_{1}\right)+\lambda_{2}\left(h_{2}\right) & =t_{1,1}+t_{1,2}+t_{1,3} \quad \text { by the first equality of }(6-4) \\
& \geq t_{2,2}+t_{1,2}+t_{1,3} \quad \text { by the first inequality of (6-5) } \\
& \geq \lambda_{1}\left(h_{1}\right)-\ell+t_{1,3} \\
& >\lambda_{1}\left(h_{1}\right)-\ell+\lambda_{1}\left(h_{2}\right)+\ell-a \\
& =\lambda_{1}\left(h_{1}\right)+\lambda_{1}\left(h_{2}\right)-a .
\end{aligned}
$$

This contradicts the inequality $\lambda_{2}\left(h_{1}\right)+\lambda_{2}\left(h_{2}\right) \leq \lambda_{1}\left(h_{1}\right)+\lambda_{1}\left(h_{2}\right)-a$ obtained in (6-13).

Suppose now that there exists $\left(t_{i, j}\right) \in \mathbb{T}\left(\lambda_{2}\right)_{\lambda_{1}}^{\nu}$ with $t_{1,2}<a-\ell$. Then we have

$$
\begin{aligned}
\lambda_{2}\left(h_{2}\right) & =t_{2,2}+t_{2,3} \quad \text { by the second equality of (6-4) } \\
& \leq t_{1,1}+t_{1,2} \quad \text { by the second inequality of (6-5) } \\
& <t_{1,1}+a-\ell=s_{1,1}+a-\ell \quad \text { by the first equality of (6-6) } \\
& \leq s_{1,1}+s_{1,3}+a-\ell .
\end{aligned}
$$

On the other hand, we have

$$
\begin{aligned}
s_{1,1}+s_{1,3}+a-\ell & =\lambda_{2}\left(h_{1}\right)+\lambda_{2}\left(h_{2}\right)-s_{1,2}+a-\ell \text { by the first equality of }(6-4) \\
& =\lambda_{2}\left(h_{1}\right)+\lambda_{2}\left(h_{2}\right)-\left(\lambda_{1}\left(h_{1}\right)-\ell\right)+a-\ell \\
& =\lambda_{2}\left(h_{1}\right)+\lambda_{2}\left(h_{2}\right)-\lambda_{1}\left(h_{1}\right)+a .
\end{aligned}
$$

Combining the two gives

$$
\lambda_{2}\left(h_{2}\right)<\lambda_{2}\left(h_{1}\right)+\lambda_{2}\left(h_{2}\right)-\lambda_{1}\left(h_{1}\right)+a \text { and so } \lambda_{1}\left(h_{1}\right)-\lambda_{2}\left(h_{1}\right)<a,
$$

which contradicts the second inequality of (6-13).

Finally suppose that there exists $\left(t_{i, j}\right) \in \mathbb{T}\left(\lambda_{2}\right)_{\lambda_{1}}^{v}$ with $t_{2,3}<a-\ell$. Then we have $\lambda_{2}\left(h_{2}\right)=t_{2,2}+t_{2,3} \leq t_{1,1}+t_{2,3}<t_{1,1}+a-\ell=s_{1,1}+a-\ell \leq s_{1,1}+s_{1,3}+a-\ell$. Since $s_{1,1}+s_{1,3}+a-\ell=\lambda_{2}\left(h_{1}\right)+\lambda_{2}\left(h_{2}\right)-\lambda_{1}\left(h_{1}\right)+a$ by (6-14), we get

$$
\lambda_{2}\left(h_{2}\right)<\lambda_{2}\left(h_{1}\right)+\lambda_{2}\left(h_{2}\right)-\lambda_{1}\left(h_{1}\right)+a,
$$

which again contradicts the second inequality of (6-13).

The following corollary is now trivially checked using Section 6.2. Thus we have proved that Theorem 1(iii) is true if $\lambda$ and $\mu$ satisfy the conditions (6-1) and (6-2) with $\boldsymbol{w}=\mathrm{id}$ or $\boldsymbol{w}=s_{2}$ (see (6-12)). 
Corollary. Keep the notation and setting in the proposition above. Let $v \in P^{+}$, and let $\ell$ be as in the proposition above. Then the assignment $\left(s_{i, j}\right) \mapsto\left(s_{i, j}^{\prime}\right)$,

$$
\begin{gathered}
s_{1,1}^{\prime}=s_{1,1}+a, \quad s_{1,2}^{\prime}=s_{1,2}-(a-\ell), \quad s_{1,3}^{\prime}=s_{1,3}+(a-\ell), \\
s_{2,2}^{\prime}=s_{2,2}+(a-\ell), \quad s_{2,3}^{\prime}=s_{2,3}-(a-\ell),
\end{gathered}
$$

defines an injective map $\mathbb{T}\left(\lambda_{2}\right)_{\lambda_{1}}^{\nu} \hookrightarrow \mathbb{T}\left(\lambda_{2}+a \omega_{1}\right)_{\lambda_{1}-a \omega_{1}}^{\nu}$.

6.4. Again, keep the notation in the proposition in 6.1. In this subsection, we prove that Theorem 1(iii) is true if $\lambda$ and $\boldsymbol{\mu}$ satisfy the conditions (6-1) and (6-2) with $\boldsymbol{w}=s_{1}$. By (6-11), it suffices to find an injective map from

$$
\mathbb{T}\left(\lambda_{2}\right)_{\lambda_{1}}^{\nu} \hookrightarrow \mathbb{T}\left(\lambda_{2}+a s_{1} \omega_{1}\right)_{\lambda_{1}-a s_{1} \omega_{1}}^{v}=\mathbb{T}\left(\lambda_{2}+a\left(\omega_{2}-\omega_{1}\right)\right)_{\lambda_{1}-a\left(\omega_{2}-\omega_{1}\right)}^{v}
$$

for each $v \in P^{+}$, where $a$ equals either 1 or $s_{1}\left(\lambda_{1}-\lambda_{2}\right)\left(h_{1}\right)$; note that

$$
s_{1}\left(\lambda_{1}-\lambda_{2}\right)\left(h_{1}\right)>0
$$

by the second equality of (6-1). This is obtained as a corollary of the following proposition.

Proposition. For each $v \in P^{+}$, there exists $\ell \geq 0$ such that for all $\left(s_{i, j}\right) \in \mathbb{T}\left(\lambda_{2}\right)_{\lambda_{1}}^{v}$,

$$
s_{1,1} \geq s_{2,2}+\ell, \quad s_{1,3} \geq a-\ell .
$$

Proof. Suppose that there exists $\left(t_{i, j}\right) \in \mathbb{T}\left(\lambda_{2}\right)_{\lambda_{1}}^{v}$ such that either $t_{1,3}=\lambda_{1}\left(h_{2}\right)$ or $t_{1,3}+t_{2,3}=\lambda_{1}\left(h_{2}\right)+t_{1,2}$. Then $\ell=0$ satisfies the condition of the proposition. Indeed, let $\left(s_{i, j}\right) \in \mathbb{T}\left(\lambda_{2}\right)_{\lambda_{1}}^{v}$. Then $s_{1,1} \geq s_{2,2}+0$ is true by the first inequality of (6-5). Also we see by the third equality of (6-6) that

$$
s_{1,3}+s_{2,3}=t_{1,3}+t_{2,3} \geq \lambda_{1}\left(h_{2}\right) .
$$

Since $s_{2,3} \leq \lambda_{2}\left(h_{2}\right)$ by (6-5), and $\lambda_{1}\left(h_{2}\right)-\lambda_{2}\left(h_{2}\right)+\lambda_{1}\left(h_{1}\right)-\lambda_{2}\left(h_{1}\right) \geq 0$ by the fact that $s_{1}\left(\lambda_{1}-\lambda_{2}\right)\left(h_{2}\right) \geq 0$ (recall that $s_{1}\left(\lambda_{1}-\lambda_{2}\right)\left(h_{2}\right) \in P^{+}$), we get

$$
s_{1,3} \geq \lambda_{1}\left(h_{2}\right)-s_{2,3} \geq \lambda_{1}\left(h_{2}\right)-\lambda_{2}\left(h_{2}\right) \geq \lambda_{2}\left(h_{1}\right)-\lambda_{1}\left(h_{1}\right) \geq a=a+0 .
$$

Consider now the case when for all $\left(t_{i, j}\right) \in \mathbb{T}\left(\lambda_{2}\right)_{\lambda_{1}}^{\nu}$, both of $t_{1,3}<\lambda_{1}\left(h_{2}\right)$ and $t_{1,3}+t_{2,3}<\lambda_{1}\left(h_{2}\right)+t_{1,2}$ hold. Since $t_{1,1} \geq t_{2,2}$ by (6-4), we can choose $\ell \geq 0$ minimal with the property that $t_{1,1} \geq t_{2,2}+\ell$ for all $\left(t_{i, j}\right) \in \mathbb{T}\left(\lambda_{2}\right)_{\lambda_{1}}^{v}$. If $\ell \geq a$, then the statement of the proposition is trivially true. Assume now that $\ell<a$, and suppose that there exists $\left(t_{i, j}\right)$ with $t_{1,3}<a-\ell$. Fix $\left(s_{i, j}\right) \in \mathbb{T}\left(\lambda_{2}\right)_{\lambda_{1}}^{v}$ such that $s_{1,1}=s_{2,2}+\ell$. Since both of $\left(s_{i, j}\right)$ and $\left(t_{i, j}\right)$ are elements of $\mathbb{T}\left(\lambda_{2}\right)^{v}$, we have, by (6-4) and (6-6),

$t_{1,2}+(a-\ell)>t_{1,2}+t_{1,3}=\lambda_{2}\left(h_{1}\right)+\lambda_{2}\left(h_{2}\right)-t_{11}=\lambda_{2}\left(h_{1}\right)+\lambda_{2}\left(h_{2}\right)-s_{11}=s_{1,2}+s_{1,3}$. 
Hence we get

$$
\begin{aligned}
\lambda_{1}\left(h_{1}\right) & \geq t_{1,2} \quad \text { by the first inequality of (6-8) } \\
& >s_{1,2}+s_{1,3}-(a-\ell) \\
& =\left(\lambda_{2}\left(h_{1}\right)+\lambda_{2}\left(h_{2}\right)-s_{1,1}\right)+\ell-a \quad \text { by the first equality of (6-4) } \\
& =\lambda_{2}\left(h_{1}\right)+\lambda_{2}\left(h_{2}\right)-s_{2,2}-a \\
& \geq \lambda_{2}\left(h_{1}\right)+\lambda_{2}\left(h_{2}\right)-\lambda_{2}\left(h_{2}\right)-a \quad \text { by the second equality of (6-4) } \\
& =\lambda_{2}\left(h_{1}\right)-a .
\end{aligned}
$$

So, $\lambda_{1}\left(h_{1}\right)>\lambda_{2}\left(h_{1}\right)-a$, which gives $a>\lambda_{2}\left(h_{1}\right)-\lambda_{1}\left(h_{1}\right)$, which is a contradiction. Hence $t_{1,3} \geq a-\ell$ for all $\left(t_{i, j}\right) \in \mathbb{T}\left(\lambda_{2}\right)_{\lambda_{1}}^{\nu}$, and the proof is complete.

The following corollary is now trivially checked using Section 6.2. Thus we have proved that Theorem 1(iii) is true if $\lambda$ and $\mu$ satisfy the conditions (6-1) and (6-2) with $\boldsymbol{w}=s_{1}$ (see (6-15)).

Corollary. Keep the notation and setting in the proposition above. Let $v \in P^{+}$, and let $\ell$ be as in the proposition above. Then the assignment $\left(s_{i, j}\right) \mapsto\left(s_{i, j}^{\prime}\right)$,

$$
\begin{gathered}
s_{1,1}^{\prime}=s_{1,1}, \quad s_{1,2}^{\prime}=s_{1,2}+(a-\ell), \quad s_{1,3}^{\prime}=s_{1,3}-(a-\ell), \\
s_{2,2}^{\prime}=s_{2,2}+\ell, \quad s_{2,3}^{\prime}=s_{2,3}+(a-\ell),
\end{gathered}
$$

defines an injective map $\mathbb{T}\left(\lambda_{2}\right)_{\lambda_{1}}^{v} \hookrightarrow \mathbb{T}\left(\lambda_{2}+a\left(\omega_{2}-\omega_{1}\right)\right)_{\lambda_{1}-a\left(\omega_{2}-\omega_{1}\right)}^{\nu}$.

\section{Acknowledgements}

The authors thank the Hausdorff Institute for Mathematics for the invitation to participate in the trimester program "On the Interaction of Representation Theory with Geometry and Combinatorics", where this collaboration was initiated. The authors would also thank Anne Schilling for helpful discussions.

\section{References}

[Bergeron et al. 2006] F. Bergeron, R. Biagioli, and M. H. Rosas, "Inequalities between LittlewoodRichardson coefficients", J. Combin. Theory Ser. A 113:4 (2006), 567-590. MR 2007f:05175 Zbl 1090.05070

[Bourbaki 2002] N. Bourbaki, Lie groups and Lie algebras: Chapters 4-6, Springer, Berlin, 2002. MR 2003a:17001 Zbl 0983.17001

[Dobrovolska and Pylyavskyy 2007] G. Dobrovolska and P. Pylyavskyy, "On products of $\mathfrak{s l}_{\mathfrak{n}}$ characters and support containment”, J. Algebra 316:2 (2007), 706-714. MR 2008j:17011 Zbl 1130.17004

[Fomin et al. 2005] S. Fomin, W. Fulton, C.-K. Li, and Y.-T. Poon, "Eigenvalues, singular values, and Littlewood-Richardson coefficients", Amer. J. Math. 127:1 (2005), 101-127. MR 2006e:05189 Zbl 1072.15010 
[Fran and McNamara 2004] c. B. Fran and P. McNamara, "Some positive differences of products of Schur functions", 2004. arXiv math.CO/0412289

[Kashiwara and Nakashima 1994] M. Kashiwara and T. Nakashima, "Crystal graphs for representations of the $q$-analogue of classical Lie algebras”, J. Algebra 165:2 (1994), 295-345. MR 95c:17025 Zbl 0808.17005

[Lam et al. 2007] T. Lam, A. Postnikov, and P. Pylyavskyy, "Schur positivity and Schur log-concavity", Amer. J. Math. 129:6 (2007), 1611-1622. MR 2009d:05255 Zbl 1131.05096

[Lascoux et al. 1997] A. Lascoux, B. Leclerc, and J.-Y. Thibon, "Ribbon tableaux, Hall-Littlewood functions, quantum affine algebras, and unipotent varieties", J. Math. Phys. 38:2 (1997), 1041-1068. MR 98c:05167 Zbl 0869.05068

[Littelmann 1994] P. Littelmann, "A Littlewood-Richardson rule for symmetrizable Kac-Moody algebras”, Invent. Math. 116:1-3 (1994), 329-346. MR 95f:17023 Zbl 0805.17019

[Littelmann 1995] P. Littelmann, "Paths and root operators in representation theory", Ann. of Math. (2) 142:3 (1995), 499-525. MR 96m:17011 Zbl 0858.17023

[Macdonald 1995] I. G. Macdonald, Symmetric functions and Hall polynomials, 2nd ed., Oxford University Press, New York, 1995. MR 96h:05207 Zbl 0824.05059

[McNamara 2008] P. R. W. McNamara, "Necessary conditions for Schur-positivity", J. Algebraic Combin. 28:4 (2008), 495-507. MR 2010a:05192 Zbl 1160.05057

[McNamara and van Willigenburg 2009] P. R. W. McNamara and S. van Willigenburg, "Positivity results on ribbon Schur function differences", European J. Combin. 30:5 (2009), 1352-1369. MR 2010i:05352 Zbl 1207.05221

[Nakashima 1993] T. Nakashima, "Crystal base and a generalization of the Littlewood-Richardson rule for the classical Lie algebras", Comm. Math. Phys. 154:2 (1993), 215-243. MR 94f:17015 Zbl 0795.17016

[Okounkov 1997] A. Okounkov, "Log-concavity of multiplicities with application to characters of $\mathrm{U}(\infty)$ ", Adv. Math. 127:2 (1997), 258-282. MR 99a:22028 Zbl 0910.22011

[Purbhoo and van Willigenburg 2008] K. Purbhoo and S. van Willigenburg, "On tensor products of polynomial representations", Canad. Math. Bull. 51:4 (2008), 584-592. MR 2009g:20102 Zbl 1192.05174

Communicated by Georgia Benkart

Received 2013-04-12 Accepted 2013-08-15

vyjayanthi.chari@ucr.edu

gfourier@math.uni-koeln.de sagaki@math.tsukuba.ac.jp
Department of Mathematics, University of California, Riverside, CA 92521, United States

Mathematisches Institut, Universität zu Köln, Germany

Department of Mathematics, University of Tsukuba, Japan 


\section{Algebra \& Number Theory}

msp.org/ant

\section{EDITORS}

MANAGING EDITOR

Bjorn Poonen

Massachusetts Institute of Technology

Cambridge, USA

\author{
EDITORIAL BOARD CHAIR \\ David Eisenbud \\ University of California \\ Berkeley, USA
}

BOARD OF EDITORS

Georgia Benkart

Dave Benson

Richard E. Borcherds

John H. Coates

J-L. Colliot-Thélène

Brian D. Conrad

Hélène Esnault

Hubert Flenner

Edward Frenkel

Andrew Granville

Joseph Gubeladze

Roger Heath-Brown

Craig Huneke

Yujiro Kawamata

János Kollár

Yuri Manin

Barry Mazur

Philippe Michel

Susan Montgomery
University of Wisconsin, Madison, USA

University of Aberdeen, Scotland

University of California, Berkeley, USA

University of Cambridge, UK

CNRS, Université Paris-Sud, France

University of Michigan, USA

Freie Universität Berlin, Germany

Ruhr-Universität, Germany

University of California, Berkeley, USA

Université de Montréal, Canada

San Francisco State University, USA

Oxford University, UK

University of Virginia, USA

University of Tokyo, Japan

Princeton University, USA

Northwestern University, USA

Harvard University, USA

École Polytechnique Fédérale de Lausanne

University of Southern California, USA
Shigefumi Mori

Raman Parimala

Jonathan Pila

Anand Pillay

Victor Reiner

Peter Sarnak

Joseph H. Silverman

Michael Singer

Vasudevan Srinivas

J. Toby Stafford

Bernd Sturmfels

Richard Taylor

Ravi Vakil

Michel van den Bergh

Marie-France Vignéras

Kei-Ichi Watanabe

Efim Zelmanov

Shou-Wu Zhang
RIMS, Kyoto University, Japan

Emory University, USA

University of Oxford, UK

University of Notre Dame, USA

University of Minnesota, USA

Princeton University, USA

Brown University, USA

North Carolina State University, USA

Tata Inst. of Fund. Research, India

University of Michigan, USA

University of California, Berkeley, USA

Harvard University, USA

Stanford University, USA

Hasselt University, Belgium

Université Paris VII, France

Nihon University, Japan

University of California, San Diego, USA

Princeton University, USA

\section{PRODUCTION}

production@msp.org

Silvio Levy, Scientific Editor

See inside back cover or msp.org/ant for submission instructions.

The subscription price for 2014 is US $\$ 225 /$ year for the electronic version, and $\$ 400 /$ year ( $\$ 55$, if shipping outside the US) for print and electronic. Subscriptions, requests for back issues and changes of subscribers address should be sent to MSP.

Algebra \& Number Theory (ISSN 1944-7833 electronic, 1937-0652 printed) at Mathematical Sciences Publishers, 798 Evans Hall \#3840, c/o University of California, Berkeley, CA 94720-3840 is published continuously online. Periodical rate postage paid at Berkeley, CA 94704, and additional mailing offices.

ANT peer review and production are managed by EditFLOw ${ }^{\circledR}$ from Mathematical Sciences Publishers.

\section{PUBLISHED BY}

mathematical sciences publishers

nonprofit scientific publishing

http://msp.org/

(C) 2014 Mathematical Sciences Publishers 


\section{Algebra \& Number Theory}

Volume $8 \quad$ No. $4 \quad 2014$

The derived moduli space of stable sheaves

Kai Behrend, Ionut Ciocan-Fontanine, Junho Hwang and Michael Rose

Averages of the number of points on elliptic curves

Greg Martin, Paul Pollack and Ethan Smith

Noncrossed product bounds over Henselian fields

Timo Hanke, DanNy NeFtin and JaCk SONN

Yangians and quantizations of slices in the affine Grassmannian

Joel Kamnitzer, Ben Webster, Alex Weekes and Oded Yacobi

Equidistribution of values of linear forms on quadratic surfaces

OLIVER SARGENT

Posets, tensor products and Schur positivity

Vyjayanthi Chari, Ghislain Fourier and Daisuke SAGaKi

Parameterizing tropical curves I: Curves of genus zero and one

DAVID E. SPEYER

Pair correlation of angles between reciprocal geodesics on the modular surface

Florin P. Boca, Vicențiu Pașol, AleXandru A. Popa and AleXandru

ZAHARESCU

Étale contractible varieties in positive characteristic

ARMin HolschbaCh, JohanNes SCHMIDT and JaKob STIX 